REVISTA MATEMÁTICA de la

Universidad Complutense de Madrid

Volumen 9, número suplementario, 1996

http://dx.doi.org/10.5209/rev_REMA.1996.v9.17516

\title{
Some selected results of professor Baltasar Rodríguez-Salinas.
}

\section{J. HORVÁTH}

\begin{abstract}
Lecture on some aspects of the research output of Baltasar Rodríguez-Salinas, given at the Meeting on Mathematical Analysis in his homage on the occasion of his seventieth birthday.
\end{abstract}

\section{Contents}

1. Asymptotic expansions, moment problem, quasi-analyticity .....2

2. Topological vector spaces....................... 12

3. Theory of measure and integration $\ldots \ldots \ldots \ldots \ldots \ldots \ldots \ldots$

References . . . . . . . . . . . . . . . . . . . . . . . . 30

The mathematical works of Baltasar Rodriguez-Salinas . . . . . . . 33

Professor Baltasar Rodríguez-Salinas Palero, whose seventieth birthday we celebrate here in Avila, published his first papers in 1942 when he was 17 years old and still in secondary school in Alcalá de Henares.

Since then he has written more than one-hundred and fifty mathematical works. It would be impossible to give an account in a one-year course of all the beautiful results with which he enriched mathematics, and it is even more so in a one-hour lecture. I will therefore present only samples which are simple to state and easy to understand, omitting of

1991 Mathematics Subject Classification: 01A70

Servicio Publicaciones Univ. Complutense. Madrid, 1996. 
necessity many of his most profound theorems. During his whole career Professor Rodriguez-Salinas has shown an uncommon ability to assimilate quickly new theories. His creativity ranges from algebra [55], [118] and projective geometry [43], [88] to mathematical economics [142], from Fourier analysis [15], [97] to oceanography [12]. In connection with the last work let me mention that he has also a degree of "ingeniero geografo". He also wrote a number of essays on the history of mathematics. From the rich variety of fields in which don Baltasar has done research, I selected three: 1) asymptotic expansions, 2) topological vector spaces, 3 ) measure and integration.

Professor Rodríguez-Salinas has written thirty joint papers, most of them since 1973. He is a generous collaborator who willingly shares his ideas with others. With one exception his nine co-authors come from the more than twenty doctoral students he has had at Zaragoza and Madrid.

In what follows, the numbers in square brackets refer to the works of Rodríguez-Salinas and the numbers in curly brackets to the works of other authors listed at the end.

\section{Asymptotic expansions, moment problem, quasi-analyticity}

Baltasar Rodríguez-Salinas wrote his doctoral dissertation [19] on differential equations under the direction of Tomás Rodríguez Bachiller in 1954. However, already at that time he was interested in questions related to asymptotic expansions whose study was initiated in Spain by Ricardo San Juan Llosa.

Before presenting the results of don Baltasar it is necessary to introduce some notation and to recall some classical facts. We shall denote by $A_{\alpha}$ the angular domain $|\theta|<\alpha \frac{\pi}{2}$ of the plane of the complex variable $z=x+i y=r e^{i \theta}$, or of the Riemann surface of the function $\log z$ if $\alpha>2$. The power series $\sum_{k \in \mathbb{N}} a_{k} z^{k}$ is an asymptotic expansion at the point $z=0$ of the function $f$ analytic in $A_{\alpha}$ if for every $n \in \mathbb{N}$ one has

$$
\left|f(z)-\sum_{k=0}^{n} a_{k} z^{k}\right|=o\left(|z|^{n}\right)
$$


as $z \rightarrow 0$ in $A_{\alpha}$. It is very important to observe that this condition has nothing to do with the convergence of the series, in spite of the fact that the initiators of the theory, T. J. Stieltjes and Henri Poincare, called them "semi-convergent series".

The function $f$ determines the coefficients $a_{k}$ but conversely distinct functions can have the same asymptotic expansion. For instance $\frac{z}{z+1}$ and $\frac{z}{z+1}+e^{-1 / z}$ defined in $A_{2}$ have $\sum_{n \in \mathbb{N}}(-1)^{n} z^{n+1}$ as asymptotic expansion at 0 . To investigate the uniqueness of the function with a given asymptotic expansion it is necessary to introduce explicit bounds for the expressions on the left hand side of (1). This leads to Watson's Problem: Let $\left(m_{n}\right)$ be a sequence of strictly positive numbers. Let $f_{1}$ and $f_{2}$ be two analytic functions in $A_{\alpha}$ and assume that there exists a sequence $\left(a_{n}\right)$ of complex numbers such that for certain constants $A>0$ and $k>0$, depending on $f_{j}$, one has

$$
\left|f_{j}(z)-\sum_{i=0}^{n-1} a_{i} z^{i}\right| \leq A k^{n} m_{n}|z|^{n}
$$

for $n \in \mathbb{N}, z \in A_{\alpha}$ and $j=1,2$. Under what condition on $\left(m_{n}\right)$ can one assert that $f_{1}(z)=f_{2}(z)$ ?

Clearly the problem is equivalent to the following: Let $\left(m_{n}\right)$ be a sequence of strictly positive numbers and $g$ an analytic function in $A_{\alpha}$ which satisfies the inequalities $|g(z)| \leq A k^{n} m_{n}|z|^{n}$ for $n \in \mathbb{N}$ and $z \in A_{\alpha}$. What conditions on $\left(m_{n}\right)$ imply that $g=0$ ?

In order to state the solution of the problem, let us introduce the function

$$
T(r)=\sup _{n \in \mathbb{N}} \frac{r^{n}}{m_{n}}, \quad r \geq 0 .
$$

Furthermore, let $\pi$ be the highest convex polygonal line below the points $\left(n, \log m_{n}\right)$ of the euclidean plane. Let $\log m_{n}^{c}$ by the $y$-coordinate of the point on $\pi$ with $x$-coordinate $n$. The sequence $\left(m_{n}^{c}\right)$ is called the logarithmically convex regularization of $\left(m_{n}\right)$ and is given also by

$$
m_{n}^{c}=\sup _{r>0} \frac{r^{n}}{T(r)}, \quad n \in \mathbb{N} .
$$

Then we have the following theorem, which for $\alpha=1$ is due to T. Carleman and A. Ostrowski and for a general $\alpha$ is the special case of a result of S. Mandelbrojt: 
The inequalities $|g(z)| \leq A k^{n} m_{n}|z|^{n}, n \in \mathbb{N}, z \in A_{\alpha}$ imply that $g(z) \equiv 0$ if and only if the following equivalent conditions are satisfied:

$$
\left(C_{\alpha}\right) \int_{0}^{\infty} \frac{\underline{\log T(r)}}{1+r^{1+\frac{1}{\alpha}}} d r=\infty, \quad \sum\left(\frac{m_{n}^{c}}{m_{n+1}^{c}}\right)^{1 / \alpha}=\infty .
$$

This solution of Watson's Problem is applied to prove the DenjoyCarleman theorem concerning quasi-analytic classes. Let again be $\left(m_{n}\right)$ a sequence of strictly positive numbers and denote by $C\left\{m_{n}\right\}$ the set of all infinitely differentiable functions $f$ in a given interval which with some constants $A>0$ and $k>0$, depending on $f$, satisfy

$$
\left|f^{(n)}(t)\right| \leq A k^{n} m_{n}
$$

for $n \in \mathbb{N}$ and every $t$. The class $C\left\{m_{n}\right\}$ is quasi-analytic if from $f_{j} \in C\left\{m_{n}\right\}, j=1,2$ and $f_{1}^{(n)}(c)=f_{2}^{(n)}(c)$ for all $n \in N$ at a point $c$ of the interval it follows that $f_{1}$ and $f_{2}$ are equal in the whole interval. The theorem in question states that $C\left\{m_{n}\right\}$ is quasi-analytic if and only if condition $\left(C_{1}\right)$ is satisfied.

It is an immediate consequence of the solution of Watson's Problem that $\left(C_{1}\right)$ is a sufficient condition. Indeed, if there exists a non-identically zero function $f \in C\left\{m_{n}\right\}$ with $f^{(n)}(c)=0$. for all $n \in \mathbb{N}$, then one can construct an infinitely differentiable, not identically zero function $g$ on $[0,1]$ such that $\left|g^{(n)}(t)\right| \leq m_{n}$ and $g^{(n)}(0)=g^{(n)}(1)=0$ for $n \in \mathbb{N}$. Consider

$$
F(\zeta)=\int_{0}^{1} g(t) e^{-t \zeta} d t
$$

Integrating by parts $n$ times we obtain

$$
F(\zeta)=\frac{1}{\zeta^{n}} \int_{0}^{1} g^{(n)}(t) e^{-t \zeta} d t
$$

and thus $|F(\zeta)| \leq m_{n}|\zeta|^{-n}$. Since $F$ is analytic for $\operatorname{Re} \zeta>0$, the change of variables $\zeta \longmapsto 1 / z$ shows that if $\left(C_{1}\right)$ is satisfied then $F=0$ and therefore $g(t) \equiv 0$.

Watson's Problem arises also in the theory of moments. Stieltjes asked. under what conditions on the sequence $\left(m_{n}\right)$ of strictly positive numbers does there exist an increasing function $\mu$ on $[0, \infty)$ such that for every $n \in \mathbb{N}$ the moment $\int_{0}^{\infty} t^{n} d \mu(t)$ is equal to $m_{n}$. Let us observe that 
a necessary condition is $m_{n}^{2} \leq m_{n-1} m_{n+1}$ for $n \geq 1$, i.e., that $m_{n}^{c}=m_{n}$ for all $n \in \mathbb{N}$.

Already Stieltjes himself gave an example of two increasing, nonequivalent functions which have all their moments equal. We are thus led to the problem of uniqueness which can be stated as follows: What conditions on $\left(m_{n}\right)$ imply that, if $\mu$ is a function of bounded variation on $[0, \infty)$ which satisfies

$$
\int_{0}^{\infty} t^{n} d \mu(t)=0 \quad \text { and } \quad \int_{0}^{\infty} t^{n} d|\mu|(t) \leq m_{n}, \quad n \in \mathbb{N},
$$

then $\mu$ is equivalent to a constant?

In order to answer this question, one introduces the Stieltjes transform of $\mu$ :

$$
F(z)=\int_{0}^{\infty} \frac{d \mu(t)}{\overline{z-t}}
$$

From the identity

$$
\frac{1}{z-t}=\frac{1}{z}+\frac{t}{z^{2}}+\cdots+\frac{t^{n-1}}{z^{n}}+\frac{t^{n}}{z^{n}(z-t)}
$$

it follows that

$$
F(z)=\frac{1}{z^{n}} \int_{0}^{\infty} \frac{t^{n} d \mu(t)}{z-t}
$$

and so

$$
|F(z)| \leq \frac{m_{n}}{|z|^{n}}
$$

in the region where $|z-t|>1$ for $0 \leq t<\infty$.

We are essentially in the case $\alpha=2$ of Watson's Problem, hence the theorem stated above implies the result due to Carleman (but contained implicitly in an earlier work of Marcel Riesz) according to which the Stieltjes moment problem is "determined". if (assuming licitly that $m_{n}=$ $\left.m_{n}^{c}\right)$

$$
\int_{1}^{\infty} \frac{\overline{\log T(r)}}{r^{\frac{3}{2}}} d r=\infty, \quad \text { or } \quad \sum\left(\frac{m_{n}}{m_{n+1}}\right)^{1 / 2}=\infty .
$$

After this long introduction I arrive at the theorems of RodríguezSalinas and the first ones I want to mention are related to the Stieltjes 
problem [17]. He proves that if $f$ is an integrable function on $(0, \infty)$ such that

$$
\int_{0}^{\infty} t^{n} f(t) d t=0
$$

and

$$
\int_{0}^{\infty} t^{n}|f(t)| d t \leq m_{n}
$$

for $n \in \mathbb{N}$ and the sequence $\left(m_{n}\right)$ satisfies condition $\left(C_{2}\right)$, then $f(t)=0$ for almost every $t$. Conversely, if

$$
\int_{1}^{\infty} \frac{\log T(r)}{r^{\frac{3}{2}}} d r<\infty
$$

then there exists a non-identically vanishing continuous function $f$ in $(0, \infty)$ which satisfies conditions (2) and (3). He proves furthermore that if instead of (2) the moments

$$
a_{n}=\int_{0}^{\infty} t^{n} f(t) d t, \quad n \in \mathbb{N},
$$

satisfy

$$
\limsup _{n \rightarrow \infty} \sqrt[n]{\left|a_{n}\right|}=\rho<\infty
$$

then (3) and $\left(C_{2}\right)$ imply that $f(t)=0$ for $t>\rho$.

Let us denote by $K\left\{m_{n} ; A_{\alpha}\right\}$ the class of analytic functions $f$ in $A_{\alpha}$ for which there exist a sequence $\left(a_{n}\right)$ of complex numbers and constants $A>0, k>0$ such that

$$
\left|f(z)-\sum_{i=0}^{n-1} a_{i} z^{i}\right| \leq A k^{n} m_{n}|z|^{n}
$$

for $z \in A_{\alpha}$ and $n \in \mathbb{N}$. Following San Juan we say that $K\left\{m_{n} ; A_{\alpha}\right\}$ is a semi-analytic class if every $f \in K\left\{m_{n} ; A_{\alpha}\right\}$ is uniquely determined by the sequence $\left(a_{n}\right)$. The theorem quoted above asserts that $K\left\{m_{n} ; A_{\alpha}\right\}$ is semi-analytic if and only if $\left(C_{\alpha}\right)$ is satisfied. As a generalization of Liouville's theorem Rodríguez-Salinas proves [44] that $K\left\{m_{n} ; A_{\alpha}\right\}$ contains only constants if and only if one has

$$
\begin{aligned}
& \lim \inf \frac{\sqrt[n]{m_{n}}}{n^{\alpha-2}}=0 \quad \text { if } \quad 0<\alpha \leq 2 \\
& \left(C_{\alpha-2}\right) \quad \text { if } \alpha>2 \text {. }
\end{aligned}
$$


San Juan and Rodriguez-Salinas considered the analogues of quasianalytic classes and of moment problems when the functions are analytic in an angular sector $A_{\alpha}$ and are not defined only on the real half-axis.

Let us denote by $C\left\{m_{n} ; A_{\alpha}\right\}$ the class of functions $f$ which are analytic in $A_{\alpha}$, have angular derivatives of all orders at the point 0 and satisfy the inequalities $\left|f^{n}(z)\right| \leq A k^{n} m_{n}$ for $z \in A_{\alpha}, n \in \mathbb{N}$ and some $A>0, k>0$, depending on $f$. We say that $C\left\{m_{n} ; A_{\alpha}\right\}$ is quasi-analytic if from $f \in C\left\{m_{n} ; A_{\alpha}\right\}$ and $f^{(n)}(0)=0$ for $n \in \mathbb{N}$ it follows that $f$ is identically zero. The theorem of Rodriguez-Salinas [44] states that $C\left\{m_{n} ; A_{\alpha}\right\}$ is quasi-analytic if and only if $\left(m_{n}\right)$ satisfies $\left(C_{\alpha+1}\right)$. For $C\left\{m_{n} ; A_{\alpha}\right\}$ to contain only constants it is necessary and sufficient to have

$$
\begin{array}{ll}
\liminf _{n \rightarrow \infty} \frac{\sqrt[n]{m_{n}}}{n^{\alpha-1}}=0 & \text { if } \quad 0<\alpha \leq 1, \\
\left(C_{\alpha-1}\right) & \text { if } \quad \alpha>1 .
\end{array}
$$

Let $f$ be an analytic function in $A_{\alpha}$ and suppose that its absolute moments on the real axis

$$
b_{n}=\int_{0}^{\infty} x^{n}|f(x)| d x
$$

satisfy an estimate $b_{n} \leq m_{n}$. San Juan, Ferrán Sunyer i Balaguer and R. P. Boas initiated the search for conditions on $\left(m_{n}\right)$ which insure that $f(z) \equiv 0$. Rodriguez-Salinas proves [21], among others, the following theorems, where the ordinary moments are denoted by

$$
a_{n}=\int_{0}^{\infty} x^{n} f(x) d x .
$$

1. Let $f$ be an analytic function in $A_{\alpha}, \alpha<1$, such that

$$
\limsup _{z \rightarrow \infty} \frac{\log |f(z)|}{|z|} \leq h<\infty .
$$

If

$$
\limsup _{n \rightarrow \infty} \frac{\sqrt[\alpha n]{\left|a_{n}\right|}}{n}=0 \text { and } \sum_{n \in \mathbb{N}} \frac{n}{\sqrt[n]{b_{n}}}=\infty,
$$

then $f(z) \equiv 0$. 
2. Let $f$ be an entire analytic function of exponential type $\leq \rho$, i.e.

$$
\limsup _{z \rightarrow \infty} \frac{\log |f(z)|}{|z|} \leq \rho .
$$

If

$$
\limsup _{n \rightarrow \infty} \frac{\sqrt[n]{\left|a_{n}\right|}}{n}=\sigma<\frac{1}{e \rho} \text { and } \sum_{n \in \mathbb{N}} \frac{n}{\sqrt[n]{b_{n}}}=\infty,
$$

then $f(z) \equiv 0$.

3. Let $f$ be an analytic function in $A_{\alpha}$ such that

$$
\limsup _{z \rightarrow \infty} \frac{\log |f(z)|}{H(|z|)} \leq h<\infty,
$$

where $H$ is a positive increasing function of $r>0$ satisfying

$$
\int_{1}^{\infty} \frac{H(r)}{r^{1+\frac{1}{\alpha}}} d r<\infty
$$

If $\left(m_{n}\right)$ is a sequence of positive numbers such that

$$
\liminf _{n \rightarrow \infty} \frac{\sqrt[a n]{m_{n}}}{n}=0
$$

and $b_{n} \leq m_{n}$, then $f(z) \equiv 0$.

4. Conversely, if

$$
\liminf _{n \rightarrow \infty} \frac{\sqrt[\alpha n]{m_{n}}}{n}>0
$$

then there exists a non identically zero bounded analytic function in $A_{\alpha}$ for which $b_{n} \leq m_{n}$.

5. Let $f$ be an analytic function in $A_{\alpha}$ such that

$$
\limsup _{z \rightarrow \infty} \frac{\log |f(z)|}{|z|^{1 / \alpha}} \leq h<\infty .
$$

If $\left(m_{n}\right)$ is a sequence of positive numbers such that

$$
\limsup _{n \rightarrow \infty} \frac{(\log n) \sqrt[a n]{m_{n}}}{\alpha n}<\frac{\pi}{2 e h}
$$

and $b_{n} \leq m_{n}$, then $f(z) \equiv 0$. 
6. Let $f$ be an analytic function of exponential type $\leq \rho$ in $A_{\alpha}$. Assume that $\left(a_{n}\right)$ satisfies the condition

$$
\begin{array}{ll}
\lim _{n \rightarrow \infty} \frac{\sqrt[\alpha n]{\left|a_{n}\right|}}{n}=0 & \text { if } \alpha<1, \\
\limsup _{n \rightarrow \infty} \frac{(\log n) \sqrt[n]{\left|a_{n}\right|}}{n}<\frac{\pi}{2 e \rho} & \text { if } \alpha=1, \\
\limsup _{n \rightarrow \infty} \sqrt[n]{\left|a_{n}\right|}<\frac{\cos \left[(2-\alpha) \frac{\pi}{2} \mid\right.}{e \rho} & \text { if } 1<\alpha \leq 2, \\
\limsup _{n \rightarrow \infty} \sqrt[n]{\left|a_{n}\right|}<\frac{1}{e \rho} & \text { if } \alpha \geq 2 .
\end{array}
$$

Let $\left(m_{n}\right)$ be a sequence of strictly positive numbers which satisfies condition $\left(C_{2}\right)$. If $b_{n} \leq m_{n}$ for $n \in \mathbb{N}$, then $f(z) \equiv 0[17]$.

7. Let $f$ be an analytic function in $A_{\alpha}$ of order less than $1 /(\alpha+2)$, i.e.

$$
|f(z)| \leq A e^{|z|^{\sigma}}
$$

for $z \in A_{\alpha}$ and some $A>0, \sigma<1 /(\alpha+2)$. Assume that

$$
\limsup _{n \rightarrow \infty} \sqrt[n]{\left|a_{n}\right|}<\infty
$$

Let $\left(m_{n}\right)$ be a sequence of positive numbers for which

$$
\liminf _{n \rightarrow \infty} \frac{\sqrt[n]{m_{n}}}{n^{\alpha+2}}=0
$$

If $b_{n} \leq m_{n}$, for $n \in \mathbb{N}$, then $f(z) \equiv 0[17]$.

8. If $\left(m_{n}\right)$ is a sequence of strictly positive numbers such that

$$
\liminf _{n \rightarrow \infty} \frac{\sqrt[n]{m_{n}}}{n^{\alpha+2}}>0
$$

then there exists a non identically zero bounded analytic function in $A_{\alpha}$ such that $a_{n}=0$ and $b_{n} \leq m_{n}$ for $n \in \mathbb{N}$ [17].

Let $C\left\{m_{n}^{(1)}\right\}$ and $C\left\{m_{n}^{(2)}\right\}$ be two quasi-analytic classes. Carleman asked whether from the hypotheses $f_{1} \in C\left\{m_{n}^{(1)}\right\}, f_{2} \in C\left\{m_{n}^{(2)}\right\}$ and 
$f_{1}^{(n)}(c)=f_{2}^{(n)}(c)$ for $n \in \mathbb{N}$ does it follow that the functions, $f_{1}$ and $f_{2}$ are identically equal. It was a triumph of Spanish mathematics when in $1936 \mathrm{San}$ Juan gave a negative answer to this question. A little later Mandelbrojt proved, using the method of San Juan, that every infinitely differentiable function is the sum of two infinitely differentiable functions which belong to quasi-analytic classes. If $f$ is not identically zero such that $f^{(n)}(c)=0$ for $n \cdot \in \mathbb{N}$ and $f=f_{1}-f_{2}$ where $f_{1}$ and $f_{2}$ belong to necessarily distinct quasi-analytic classes then $f_{1}^{(n)}(c)=f_{2}^{(n)}(c)$ for $n \in \mathbb{N}$ but $f_{1} \neq f_{2}$. In his doctoral dissertation $\{2\}$ Th. Bang improved Mandelbrojt's theorem by proving the following result: Let $C\left\{m_{n}^{(1)}\right\}$ and $C\left\{m_{n}^{(2)}\right\}$ be two classes of infinitely differentiable functions defined on the whole real line. In this case one may assume that each sequence $\left(m_{n}^{(j)}\right)$ is logarithmically convex. Let $\pi_{j}$ be the convex polygonal line with vertices $\left(n, \log m_{n}^{(j)}\right)$. Every function of $C\left\{m_{n}^{(1)}+m_{n}^{(2)}\right\}$ is the sum of a function in $C\left\{m_{n}^{(1)}\right\}$ and of a function in $C\left\{m_{n}^{(2)}\right\}$ if and only if the highest minorant of $\pi_{1}$ and $\pi_{2}$ is convex.

San Juan and Rodriguez-Salinas found analogues of Bang's theorem for semi-analytic classes. In particular, don Baltasar studied the case of angular domains [44]. For two sequences $\left(\nu_{n}^{(1)}\right)$ and $\left(\nu_{n}^{(2)}\right)$ of strictly positive numbers he introduced the following hypotheses:

$\left(H_{1}\right)$ The sequences $\left(\nu_{n}^{(1)}\right)$ and $\left(\nu_{n}^{(2)}\right)$ are logarithmically convex.

$\left(\mathrm{H}_{2}\right)$ The highest minorant of the two polygonal lines, which have the points $\left(n, \log \nu_{n}^{(1)}\right)$ and $\left(n, \log \nu_{n}^{(2)}\right)$ as respective vertices, is convex.

Then we have:

1. Case $\alpha=2$. Let $m_{n}=m_{n}^{(1)}+m_{n}^{(2)}$. If $\left(m_{n}\right)$ does not satisfy $\left(C_{2}\right)$ and the sequences $\left(m_{n}^{(1)}\right)$ and $\left(m_{n}^{(2)}\right)$ satisfy $\left(H_{1}\right)$ and $\left(H_{2}\right)$ then there exist two distinct analytic functions $f_{1}$ and $f_{2}$ belonging to the classes $K\left\{m_{n}^{(1)} ; A_{2}\right\}$ and $K\left\{m_{n}^{(2)} ; A_{2}\right\}$, respectively, which have the same asymptotic expansion at 0 .

2. Case $0<\alpha<2$. Let $0 \leq \delta \leq 2-\alpha$ and $m_{n}=m_{n}^{(1)}+m_{n}^{(2)}$. Assume that $\left(m_{n}\right)$ does not satisfy $\left(C_{\alpha}\right)$ and that the sequences $\left(n^{\delta n} m_{n}^{(1)}\right)$ and $\left(n^{\delta n_{n}} m_{n}^{(2)}\right)$ satisfy the hypotheses $\left(H_{1}\right)$ and $\left(H_{2}\right)$. Then one can find functions $f_{1} \neq f_{2}$ belonging to $K\left\{m_{n}^{(1)} ; A_{2}\right\}$ and $K\left\{m_{n}^{(2)} ; A_{2}\right\}$, respectively, which have the same asymptotic expansion at 0 .

Rodriguez-Salinas has a theorem also in the case $\alpha>2$ and results about regions which are not angular sectors. 
Bang asked whether there can exist two distinct functions belonging to two quasi-analytic classes which coincide in a whole interval and are analytic there. Rodriguez-Salinas gave a positive answer to this question [30]. He proves first the following theorem:

Let $\left(m_{n}^{(1)}\right)$ and $\left(m_{n}^{(1)}\right)$ be two sequences of strictly positive numbers, $\alpha>0$, and $m_{n}=\left(m_{n}^{(1)}+m_{n}^{(1)}\right) /(\alpha n) !$ where $(\alpha n) !=\Gamma(\alpha n+1)$. Assume that $\left(m_{n}\right)$ does not satisfy $\left(C_{1}\right)$ and that the sequences $\left(m_{n}^{(1)} /(\alpha n) !\right)$ and $\left(m_{n}^{(2)} /(\alpha n) !\right)$ satisfy $\left(H_{1}\right)$ and $\left(H_{2}\right)$. Then there exists a pair of functions $f_{1} \neq f_{2}$ defined for $-\infty<t<\infty$, analytic for $t \neq 0$, equal for $t<0$ and belonging to the classes $C\left\{m_{n}^{(1)}\right\}$ and $C\left\{m_{n}^{(2)}\right\}$, respectively.

Then he shows that the bounds $m_{n}^{(j)}(j=1,2)$ can be chosen in such a way that the classes $C\left\{m_{n}^{(j)}\right\}$ are quasi-analytic.

It was also Carleman who posed the problem of equivalence of classes of infinitely differentiable functions, that is, to give conditions on $\left(m_{n}\right)$ and $\left(\tilde{m}_{n}\right)$ which ensure that $C\left\{m_{n}\right\}=C\left\{\tilde{m}_{n}\right\}$. The solution was given in 1940 by Henri Cartan and Mandelbrojt who introduced for this purpose new kinds of regularizations of sequences, distinct from the logarithmically convex regularization and depending on the type of interval on which the functions are defined $\{19\}$. For instance, in the case of a finite open interval they introduce the function

$$
S(r)=\max _{n \leq r} \frac{r^{n}}{m_{n}}, \quad r \geq 0
$$

and define the exponential regularization of $\left(m_{n}\right)$ through the intermediary of logarithms by

$$
m_{n}^{\circ}=\sup _{r \geq n} \frac{r^{n}}{S(r)}, \quad n \in \mathbb{N}
$$

Rodríguez-Salinas considered [27] the same problem for the classes $K\left\{m_{n} ; A_{\alpha}\right\}$ and later for classes $K\left\{m_{n} ; \Omega\right\}$ of analytic functions defined in certain general regions $\Omega$ and satisfying inequalities of the type (4). He announced his result in 1961 in a four page long note [36] but published the details only in 1987 in six articles [107]-[112] whose total length is 89 pages. To obtain his theorem Rodríguez-Salinas had to introduce an even more refined kind of regularization and study questions related to conformal representation and to the bounds of derivatives of polynomials. Here is the statement of the theorem: 
Let $\Omega$ be a bounded connected set in the complex plane which consists of more than one point and such that the origin 0 belongs to the boundary $\partial \Omega$ of $\Omega$. Let $B$ be the unbounded component of $C \bar{\Omega}$ and $w=$ $w(z)$ the conformal representation of $B$ onto the exterior $\{w:|w|>1\}$ of the unit disk such that $w(\infty)=\infty$ and $\lim _{z \rightarrow \infty} w^{\prime}(z)=c_{0}>0$. Define the functions

$$
\sigma(r)=\sup _{|z|=r} \log |w(z)|
$$

and

$$
\varphi(t)=\inf _{r>0} \frac{e^{t \sigma(r)}}{r}
$$

For $\rho>0$ let $\Omega_{\rho}$ be the component of $\Omega \cap\{z:|z|<\rho\}$ which contains 0 on its boundary and let $w_{\rho}, \sigma_{\rho}, \varphi_{\rho}$ be the analogous functions corresponding to $\Omega_{\rho}$.

Suppose that there exist two constants $b>a \geq 1$ such that $a \leq \sigma(2 r) / \sigma(r) \leq b$ for $0<r \leq \sup \{|z|: z \in \Omega\}$. The point 0 is said to be semi-regular if there exist two positive numbers $\lambda$ and $\rho_{0}$ such that $\sigma_{\rho}(r) \leq \lambda \sigma(r) / \sigma(\rho)$ for $r \leq \rho \leq \rho_{0}$. The point 0 is regular if there exists furthermore a sequence $\left(P_{k}(z)\right)$ of polynomials such that $\left|P_{k}(z)\right| \leq 1$ for $z \in \Omega$ and

$$
\frac{\left|P_{k}^{(n)}(0)\right|}{n !} \geq q^{n} \varphi\left(\frac{k}{n}\right)^{n}
$$

for $k / h \geq n \geq h$, where $0<q \leq 1$ and $h=h(\Omega)$ is sufficiently large.

The regularization $\left(\log m_{n}^{*}\right)$ of $\left(\log m_{n}\right)$ by means of $\log \varphi(t)$ is defined by

$$
S(t)=\max _{n \leq t} \frac{\varphi\left(\frac{t}{n}\right)^{n}}{m_{n}}
$$

and

$$
m_{n}^{*}=\sup _{t \geq n} \frac{\varphi\left(\frac{t}{n}\right)^{n}}{S(t)}
$$

If 0 is a regular point, then $K\left\{m_{n} ; \Omega\right\}=K\left\{\tilde{m}_{n} ; \Omega\right\}$ if and only if there exist two positive constants $a$ and $b$ such that

$$
a^{n} \leq \frac{\tilde{m}_{n}^{*}}{m_{n}^{*}} \leq b^{n} \quad \text { for } n \in \mathbb{N}
$$

Rodríguez-Salinas has a similar result for unbounded domains. 
To conclude this section I would like to mention without details three other contributions of don Baltasar:

1. Convexity inequalities for the bounds of the derivatives [23] and for the coefficients and bounds of asymptotic expansions [24] of a function analytic in $A_{\alpha}$.

2. A generalization of a Phragmén-Lindelöf type theorem of $\mathrm{L}$. Ahlfors and M. Heins [16].

3. An analogue for several variables of the Denjoy-Carleman theorem on quasi-analytic classes [22].

\section{Topological vector spaces}

Following the terminology introduced by A. Grothendieck $\{12\}$, a Banach space $E$ is said to have the Dieudonné property if for every $\mathrm{Ba}$ nach space $F$ every continuous linear map $T: E \rightarrow F$, which maps sequences that are Cauchy for the weakened topology $\sigma\left(E, E^{\prime}\right)$ into $\sigma\left(F, F^{\prime}\right)$-convergent sequences, also maps bounded subsets of $E$ into $\sigma\left(F, F^{\prime}\right)$-compact subsets of $F$ (i.e. $T$ is weakly compact).

Rodríguez-Salinas obtains [101] a large class $\mathcal{C}$ of Banach spaces such that if $E \in \underset{\sim}{\mathcal{C}}$, then the space $\mathcal{C}(X ; E)$ of continuous functions defined on the compact space $X$ with values in $E$, equipped with the topology of uniform convergence, has the Dieudonné property. To define $\underset{\sim}{\mathcal{C}}$ he says that $E$ has property $(A)$ if for some norm equivalent to the original norm on $E$ there exists a Suslin subspace $B_{0}$ of $E^{\mathbb{N}}$, consisting of Cauchy sequences for $\sigma\left(E, E^{\prime}\right)$, such that for each $e^{\prime} \in E^{\prime}$ there exists $\left(e_{n}\right) \in B_{0}$ with $\left\|e_{n}\right\| \leq 1+\frac{1}{n}$ for $n \in \mathbb{N}$ and $\lim _{n \rightarrow \infty}\left\langle e^{\prime}, e_{n}\right\rangle=\left\|e^{\prime}\right\|$.

The space $E$ belongs to $\underset{\sim}{\mathcal{C}}$ if every separable subspace of $E$ is contained in another separable subspace which has property (A).

If the topological dual $E^{\prime}$ of $E$ is separable, then $E$ belongs to $\mathcal{C}$. This implies a theorem of Fernando Bombal and Pilar Cembranos $\{\tilde{5}\}$ according to which if $E^{\prime}$ is separable, then $\mathcal{C}(X ; E)$ has the Dieudonné property.

The class $\underset{\sim}{\mathcal{C}}$ has strong stability properties:

1. If $E_{k} \in \underset{\sim}{\mathcal{C}}$ for $1 \leq k \leq n$, then $\prod_{k=1}^{n} E_{k} \in \mathcal{C}$.

2. If $F$ is the image of $E \in \underset{\sim}{\mathcal{C}}$ under a surjective continuous linear 
map, then $F \in \underset{\sim}{\mathcal{C} \text {. }}$

N. Bourbaki introduced barrelled locally convex spaces to characterize those spaces for which the principle of uniform boundedness holds. It is a simple but important result that every Baire locally convex space is barrelled. Later new classes between Baire and barrelled spaces were introduced with the purpose to find new closed graph theorems, stability theorems, and to distinguish between $(L F)$-spaces. Most of them are discussed in the Research Note of Michael Kunzinger $\{16\}$.

A locally convex space $E$ is said to be suprabarnelled by Manuel Valdivia $\{24\}$ and a $(d b)$-space by W. J. Robertson, I. Tweddle and F. E. Yeomans $\{21\}$ if it satisfies the following condition: If $\left(E_{k}\right)$ is an increasing sequence of subspaces of $E$ such that $\cup_{k \in \mathbb{N}} E_{k}=E$, then there exists $p \in \mathbb{N}$ such that $E_{p}$ (hence also $E_{k}$ for $k \geq p$ ) is dense in $E$ and $\underline{b}$ arrelled.

Rodríguez-Salinas [80] refined this classification. He says that a barrelled space is barrelled of class 0 . Then he defines barrelled spaces of class $\alpha \geq 1$ by transfinite induction. If $\alpha$ has a predecessor, then $E$ is barrelled of class $\alpha$ if, given an increasing sequence $\left(E_{k}\right)$ of subspaces of $E$ such that $\cup_{k \in \mathbb{N}} E_{k}=E$, there exists $p \in \mathbb{N}$ such that $E_{\mathrm{p}}$ is dense in $E$ and barrelled of class $\alpha-1$. Thus the suprabarrelled spaces are precisely the barrelled spaces of class 1 . If $\alpha$ is a limit ordinal then $E$ is barrelled of class $\alpha$ if it is barrelled of class $\beta$ for every $\beta<\alpha$.

For a finite $n$ there is a direct description of barreledness of class $n$ [81], $\{17\}$ which establishes a remarkable link with $M$. de Wilde's spaces with webs. An $n$-web is a collection $\left\{E_{k_{1} \ldots k_{p}}\right\}$ of subspaces of $E$ where $k_{j} \in \mathbb{N}$ for $1 \leq j \leq p$ and $1 \leq p \leq n$, such that $\left(E_{k}\right)$ is an increasing cover of $E$ and for each $(p-1)$-tuple $k_{1}, \ldots, k_{p-1} \quad(2 \leq p \leq n)$ the sequence $\left(E_{k_{1}, \ldots, k_{p-1} k}\right)_{k}$ is an increasing cover of $E_{k_{1}, \ldots, k_{p-1}}$. The space $E$ is barrelled of class $n$ if given an $n$-web there exists some $n$-tuple $r_{1}, \ldots, r_{n}$ of integers such that $E_{r_{1}, \ldots, r_{n}}$ is dense in $E$ and barrelled.

This description leads to superbarrelled spaces introduced by don Baltasar in a recent article [146]. A web is a family $\left\{E_{k_{1}, \ldots, k_{p}}\right\}$ of subspaces of $E$ as above, except that now $p$ may take any integral value $\geq 1$. The space $E$ is superbarrelled if given a web $\left\{E_{k_{1}, \ldots, k_{p}}\right\}$ there exists a sequence $\left(r_{p}\right)$ of integers such that each subspace $E_{r_{1}, \ldots, r_{p}}$ is dense in $E$ and barrelled. He proves that a superbarrelled space is barrelled of class $\alpha$ for every $\alpha$, and that a barrelled space is superbarrelled if and only if 
it is barrelled of class $\omega_{1}$, where $\omega_{1}$ is the first uncountable ordinal.

On the other hand, $E$ is the totally barrelled $\{25\}$ (or $\sigma$-barrelled in the terminology of don Baltasar [84]) if, given a sequence $\left(E_{k}\right)$ of subspaces of $E$ such that $\cup_{k \in \mathbb{N}} E_{k}=E$, there exists $p \in \mathbb{N}$ such that $E_{p}$ is barrelled and its closure $E_{p}$ has finite codimension in $E$. We have the following implications for $\alpha>\beta \geq 1$ :

Baire $\Rightarrow$ totally barrelled $\Rightarrow$ superbarrelled $\Rightarrow$ barrelled of class $\alpha \Rightarrow$ barrelled of class $\beta \Rightarrow$ suprabarrelled $\Rightarrow$ barrelled.

Let $\Sigma$ be a $\sigma$-algebra of subsets of some set $X$. Denote by $\ell_{0}^{\infty}(X, \Sigma)$ the vector space generated by the characteristic functions $\chi_{A}$ of the sets $A$ belonging to $\Sigma$, equipped with the maximum-norm $\|f\|=\max _{x \in X}|f(x)|$. If $X=\mathbb{N}$ and $\Sigma$ is the $\sigma$-algebra $2^{X}$ of all subsets of $\mathbb{N}$, then one usually writes $\ell_{0}^{\infty}$ instead of $\ell_{0}^{\infty}\left(\mathbb{N}, 2^{N}\right)$. J. Dieudonné proved that $\ell_{0}^{\infty}$ is barrelled and Grothendieck that so is $\ell_{0}^{\infty}(X, \Sigma)$. Valdivia $\{23\}$ proved that $\ell_{0}^{\infty}(X, \Sigma)$ is suprabarrelled and Rodriguez-Salinas [81] that it is barrelled of class 2. J. C. Ferrando and Manuel López Pellicer $\{9\},\{17\},\{11\}$ proved that $\ell_{0}^{\infty}(X, \Sigma)$ is barrelled of class $n$ for every $n \in \mathbb{N}$, and Rodríguez-Salinas points out in [146] that López Pellicer actually proved in $\{18\}$ that $\ell_{0}^{\infty}(X, \Sigma)$ is superbarrelled. However, J. Arias de Reyna $\{1\}$ proved that $\ell_{0}^{\infty}(X, \Sigma)$ is not totally barrelled.

A locally convex space $F$ is said to be $\Gamma_{r}$ by Valdivia and infra-s by $N$. Adasch if it has the following property: For every barrelled space $E$ every linear map $T: E \longrightarrow F$, whose graph is closed, is continuous. These authors gave various intrinsic characterizations of $\Gamma_{r}$-spaces $\{15\}$. Rodríguez-Salinas [80] calls the $\Gamma_{r}$-spaces also $\Gamma_{r}^{(0)}$-spaces and presents the following inductive definition: If the ordinal number $\alpha \geq 1$ has a predecessor, then a $\Gamma_{r}^{(\alpha)}$-space is a union of an increasing sequence of $\Gamma_{r}^{(\alpha-1)}$-subspaces. If $\alpha$ is a limit ordinal, then $F$ is a $\Gamma_{r}^{(\alpha)}$-space provided that it is a $\Gamma_{r}^{(\beta)}$-space for some $\beta<\alpha$. He then proves the following theorem: Let $E$ be a barrelled space of class $\alpha$ and $F$ a $\Gamma_{r}^{(\alpha)}$-space. If $T: E \rightarrow F$ is a linear map with closed graph, then $T$ is continuous and there exists a subspace $F_{0}$ of $F$ such that $F_{0}$ is a $\Gamma_{r}$-space and $T(E) \subset F_{0}$.

Analogously to the above definition, given a fixed locally convex space $E$ one says that $F$ is a $\Gamma_{r}(E)$-space if every linear map $T: E \rightarrow F$ with closed graph is continuous. Rodriguez-Salinas says [80] that the linear map $T: E \rightarrow F$ is subcontinuous if for every series $\sum_{k \in \mathbb{N}} x_{k}$ in $E$ 
which is subseries convergent (i.e. for every strictly increasing sequence $\left(k_{j}\right)_{j \in \mathbb{N}}$ of positive integers the series $\sum_{j \in \mathbb{N}} x_{k_{j}}$ converges) the series $\sum_{k \in \mathbb{N}} T x_{k}$ converges unconditionally in $F$ and one has $T\left(\sum_{k \in \mathbb{N}} x_{k}\right)=$ $\sum_{k \in \mathbb{N}} T x_{k}$. He obtains, among others, the following theorems:

1. Let $E$ and $F$ be two locally convex spaces. Assume that $F$ is sequentially complete, is a $\Gamma_{r}\left(\ell_{0}^{\infty}\right)$-space and does not contain a subspace isomorphic to $\ell^{\infty}$. If $T: E \rightarrow F$ is a linear map with closed graph, then $T$ is subcontinuous.

2. Let $E$ and $F$ be two locally convex spaces and let $\left(F_{k}\right)$ be an increasing sequence of subspaces of $F$ such that $\cup_{k \in \mathbb{N}} F_{k}=F$. Assume that for every $k \in \mathbb{N}$ there exists a topology $\mathcal{T}_{k}$ on $F_{k}$, finer than the topology induced by $F$ and such that $F_{k}$ equipped with $\mathcal{T}_{k}$ is a sequentially complete space, is a $\Gamma_{r}$-space and contains no subspace isomorphic to $\ell^{\infty}$. If $T: E \rightarrow F$ is a linear map with closed graph, then there is a $p \in \mathbb{N}$ such that $T(E) \subset F_{p}$ and the map $T: E \rightarrow F\left[\mathcal{T}_{p}\right]$ is subcontinuous.

The beautiful proofs employ the map $S: \ell_{0}^{\infty} \rightarrow E$ defined by $\left(a_{k}\right) \mapsto \Sigma a_{k} x_{k}$ for a given subseries convergent series $\Sigma x_{k}$. If $F$ is a $\Gamma_{r}\left(\ell_{0}^{\infty}\right)$-space, then $T \circ S$ is continuous and $m(A)=T\left(S\left(\chi_{A}\right)\right)$ is a bounded measure with values in $F$ to which the theorem of J. Diestel and Barbara Faires $\{8\}$ can be applied. Inspired by these results, Ferrando and López Pellicer $\{10\}$ proved another closed graph theorem whose conclusion is that a certain linear map is subcontinuous.

De Wilde's theory of spaces with $\mathcal{C}$-webs is widely known nowadays $\{15\}$. One of the basic theorems of de Wilde states that if $E$ is a Fréchet space or an ultrabornological space and $F$ a space with a $\mathcal{C}$-web, then every linear map $E \rightarrow F$ with closed graph is continuous. RodríguezSalinas [85] shows that if $E$ is a metrizable totally barrelled space and the $\mathcal{C}$-web on $F$ is absolutely convex, then the same conclusion holds.

The Austrian mathematician E. Helly was the first to prove the theorem on the extension of linear forms which now bears the name of $H$. Hahn and St. Banach $\{7\}$. He used in his proof the following fact, the particular case for $n=1$ of his famous theorem on convex sets: If $\left[a_{\alpha}, b_{\alpha}\right]$ is a family of finite closed intervals in $\boldsymbol{R}$, such that any two have a point in common, then $\dot{n}_{\alpha}\left[a_{\alpha}, b_{\alpha}\right] \neq 0$. Replacing the intervals by balls, $L$. Nachbin characterized the real Banach spaces $F$ which have the following property: Given an arbitrary real Banach space $E$, a linear subspace 
$E_{0}$ of $E$ and a continuous linear map $f_{0}: E_{0} \rightarrow F$, there exists a linear map $f: E \rightarrow F$ such that $f(x)=f_{0}(x)$ for $x \in E_{0}$ and $\|f\|=\left\|f_{0}\right\|$. A. W. Ingleton proved an analogous theorem for ultrametric normed spaces. In this case the condition can be stated as follows: Every family of closed balls, totally ordered by inclusion, has a non-empty intersection. Indeed, in an ultrametric space if two balls have a point in common then one is contained in the other. In collaboration with L. Bou Garcia [64] don Baltasar proved a general principle from which the three mentioned results follow easily:

Let $E$ and $F$ be two vector spaces over an arbitrary commutative field $K$. Let $\mathcal{A}$ be a non-empty collection of subsets of $F$ which has the following properties:

a) $\mathcal{A}$ is invariant with respect to translations;

b) if $\mathcal{B}$ is a collection of sets belonging to $\mathcal{A}$, such that two sets in $\mathcal{B}$ have a point in common, then the intersection of all the sets belonging to $B$ is not empty.

Let $\psi$ be a map from $E$ into $\mathcal{A}$ such that 1) $\psi(x+y) \subset \psi(x)+\psi(y)$ for $x, y \in E, 2) \psi(\alpha x)=\alpha \psi(x)$ for $\alpha \in K, x \in E$. Let $E_{0}$ be a subspace of $E$ and $f_{0}: E_{0} \rightarrow F$ a linear map such that $f_{0}(x) \in \psi(x)$ for each $x \in E_{0}$. Then there exists a linear map $f: E \rightarrow F$ such that $f(x)=f_{0}(x)$ for $x \in E_{0}$ and $f(x) \in \psi(x)$ for every $x \in E$.

Rodríguez-Salinas was very interested in the problem of extension of linear maps. He wrote an excellent expository account [61] which summarizes his own research and that of others, and has a very extensive list of references. He lectured on it in 1966 in Namur [52] and wrote a monograph [60] on the connection between the extension of additive maps and of measures. I would like to relate one of his numerous results which is interesting also from the point of view of abstract algebra [41].

Let $X$ be an additively written commutative group. A real-valued function $f$ defined on a subgroup $D(f)$ of $X$ is additive in $X$ if $f(x+y)=$ $f(x)+f(y)$ for $x, y \in D(f)$. A function $p: X \rightarrow \boldsymbol{R}$ is subadditive on $X$ if $p(x+y) \leq p(x)+p(y)$ for $x, y \in X$. A set $P$ of subadditive functions on $X$ is a $\left(Z_{1}\right)$ set if for every totally ordered subset $P_{0}$ of $P$ the function $\inf \left\{p: p \in P_{0}\right\}$ also belongs to $P$.

If $f_{1}$ and $f_{2}$ are two additive functions in $X$ with domains $D\left(f_{1}\right)$ and $D\left(f_{2}\right)$, we write $f_{1} \subset f_{2}$ if $D\left(f_{1}\right) \subset D\left(f_{2}\right)$ and $f_{1}(x)=f_{2}(x)$ for every $x \in D\left(f_{1}\right)$. A set $F$ of additive functions in $X$ is a $\left(Z_{2}\right)$ set if for every 
subset $F_{0}$ of $F$, which is totally ordered with respect to $C$, the least upper bound of $F_{0}$ belongs to $F$. Given a set $P$ of subadditive functions on $X$ and an additive function $f$ in $X$, we denote by $P(f)$ the set of all the functions $p \in P$ such that the function $f[p]$ defined by

$$
f[p](x)=\inf _{y \in D(f)}[p(x+y)-f(y)]
$$

belongs to $P$.

Let now $P$ be a $\left(Z_{1}\right)$ set of subadditive functions on $X$ and $F$ a $\left(Z_{2}\right)$ set of additive functions in $X$. If $p_{0} \in P, f_{0} \in F$ and $p_{0} \in P\left(f_{0}\right)$, then there exist functions $p \in P$ and $f \in F$ such that

1. a) $p \leq p_{0}$,

b) if $q \in P\left(f_{0}\right)$ and $q \leq p$, then $q=p$.

2. a) $f_{0} \subset f$,

b) $f(y)=p(x+y)-p(y)$ for $x \in X, y \in D(f)$,

c) if $g \in F, p \in P(g)$ and $f \subset g$, then $g=f$.

From this abstract extension theorem Rodríguez-Salinas deduces the following Hahn-Banach theorem for modules. Let $A$ be a not necessarily commutative ring with unit element and $r: A \rightarrow \boldsymbol{R}$ a non-constant homomorphism of rings. Let $X$ be a left $A$-module. An additive function $f$ in $X$ defined on a submodule $Y$ of $X$ is $A$-modular if $f(\alpha x)=r(\alpha) f(x)$ for $\alpha \in A, x \in Y$. We chose an $(r)$-generating semi-ring $A^{+}$, i.e. a subset $A^{+}$of $A$ such that $A^{+}+A^{+} \subset A^{+}, A^{+}, A^{+} \subset A^{+}, r\left(A^{+}\right) \subset(0, \infty)$ and $A=A^{+}-A^{+}$. A subadditive function $p$ on $X$ is $A^{+}$-semimodular if $p(\alpha x) \leq r(\alpha) p(x)$ for $\alpha \in A^{+}, x \in X$.

Theorem Let $P$ be the set of all $A^{+}$-semimodular subadditive functions on $X$ and $F$ the set of all $A$-modular additive functions in $X$. If $p_{0} \in P$ and $f_{0} \in F$ satisfy $f_{0}(x) \leq p_{0}(x)$ for $x \in D\left(f_{0}\right)$, then there exist $p \in P$ and $f \in F$ such that:

1. $p \leq p_{0}$ and $f_{0} \subset f$;

2. $\cdot f(y)=p(x+y)-p(y)=p(y)$ for $x \in X, y \in D(f)$;

3. if $q \in P$ satisfies $f_{0}(x) \leq q(x)$ for every $x \in D\left(f_{0}\right)$ and $q \leq p$, then $q=p$ 
4. if $g \in F$ satisfies $g(x) \leq p(x)$ for every $x \in D(g)$, then $g \subset f$.

We are thus faced with the problem to know when is $D(f)$ the whole group $X$. To answer this question Rodríguez-Salinas introduces a generalization of solvable groups. He says that a group $G$ is perfect if given a finite family $\left\{\alpha_{1}, \ldots, \alpha_{m} ; \beta_{1}, \ldots, \beta_{n}\right\}$ of elements of $G$ there exists another finite family $\left\{\xi_{1}, \ldots, \xi_{r} ; \eta_{1}, \ldots, \eta_{s}\right\}$ and a bijective map $\varphi$ from $\{(\rho, \mu): 1 \leq \rho \leq r, 1 \leq \mu \leq m\}$ onto $\{(\sigma, \nu) ; 1 \leq \sigma \leq s, 1 \leq \nu \leq n\}$ such that for $\varphi(\rho, \nu)=(\sigma, \nu)$ one has $\xi_{\rho} \alpha_{\mu}=\eta_{\sigma} \beta_{\nu}$. Obviously every abelian group is perfect. A group $G$ is accessible if it has a, possibly transfinite, normal series $\left(G_{\lambda}\right)$ such that all the quotient groups are perfect. With this definition don Baltasar proves that $D(f)=X$ if there exists a multiplicative subgroup $G$ of $A^{+}$which satisfies the following conditions:

1. $G$ is an accessible group;

2. Every element $a \in A^{+}$can be written in the form $a=\sum_{i=1}^{n} c_{i} \alpha_{i}$, where the $c_{i}$ belong to the center of $A^{+}$and $\alpha_{i} \in G$.

I have neither the time nor the competence to report on the papers don Baltasar has written in collaboration with Francisco L. Hernández [113], [117], [128], [131], [139] on the geometry of Orlicz spaces. Fortunately they have published an expository lecture [120] which summarizes some of their results.

The subdivision in three parts of this lecture is artificial since many concepts discussed in this section have a close relation to measure theory. For instance, the algebraic dual of $\ell_{0}^{\infty}(X, \Sigma)$ is identified with the space of finitely additive measures $m$ defined on $\Sigma$ by means of $m(A)=\left\langle f, \chi_{A}\right\rangle$ for $f \in \ell_{0}^{\infty}(X, \Sigma)^{*}$ and $A \in \Sigma$. Rodríguez-Salinas [60] investigates together with the extension of linear maps also the extension of a measure defined on a Boole algebra.

The Dieudonné-Grothendieck theorem $\{8\}$ states that if $m: \Sigma \rightarrow F$ is a measure defined on a $\sigma$-algebra $\Sigma$ of subsets of a set $X$ with values in a Banach space $F$ and if for $x^{\prime}$ belonging to a total subset of $F^{\prime}$ the scalarvalued measures $x^{\prime} \circ m$, defined by $A \mapsto\left\langle m(A), x^{\prime}\right\rangle$, are bounded and finitely additive, then $m$ is a bounded vector measure. Rodríguez-Salinas [82] proves that the theorem is true if and only if $F$ is a $\Gamma_{r}\left(\ell_{0}^{\infty}(x, \Sigma)\right)$ space. 


\section{Theory of measure and integration}

This is the largest and possibly the most important part of the oeuvre of Baltasar Rodriguez-Salinas, and I will be able to cover only a very small portion of it. Furthermore, these works consist mainly of great theories and it is difficult to single out isolated theorems. I will concentrate on describing briefly two of these theories: 1) Radon measures of type $(\mathcal{H})$, and 2) Integration of functions with values in a locally convex space.

1) Until about 1964 there were essentially two theories of measure: 1. Abstract measures as discussed e.g. in the book of Paul Halmos $\{13\}$, and 2. Radon measures on locally compact spaces developed by Henri Cartan and André Weil, and codified in the book by N. Bourbaki $\{6\}$. It became, however, apparent that, mainly because of their applications to probability theory, it is necessary to define Radon measures also on spaces that are not locally compact, e.g. on infinite-dimensional topological vector spaces. In 1964 Laurent Schwartz first in a series of lectures in Lisbon and then in a course at the Paris University developed a theory of Radon measures on arbitrary topological spaces $\{22\}$. In his definition a Radon measure on a topological space $E$ is an abstract measure $\mu$ which, among others, has the property that $\mu(K)<\infty$ for every compact subset $K$ of $E$. Thus the counting measure $\mu$ on $\boldsymbol{R}$, for which $\mu(A)$ is the number of elements in $A$ if $A$ is finite and $\mu(A)=\infty$ if $A$ is infinite, is not a Radon measure in the sense of Schwartz.

Rodríguez-Salinas introduced in collaboration with Pedro Jiménez Guerra the concept of Radon measures of type $(\mathcal{H})$ [62], [68], [69], [76]. Let $E$ be a topological space and $\mu$ a positive measure on $E$, i.e. a set function defined on a $\sigma$-algebra $\Sigma$ of subsets of $E$ such that $0 \leq \mu(A) \leq$ $\infty$ for $A \in \Sigma$ and $\mu\left(\cup_{n} A_{n}\right)=\Sigma_{n} \mu\left(A_{n}\right)$ if $\left(A_{n}\right)$ is a sequence of pairwise disjoint sets in $\Sigma$. Assume that $\Sigma$ contains the $\sigma$-algebra $B$ of Borel subsets of $E$. A subset $K$ of $E$ is said to be $\mu$-compact if given a cover $\left(G_{\lambda}\right)_{\lambda \in \Lambda}$ of $K$ by open sets and $\epsilon>0$, there exists a finite subset $\Phi$ of the index set $\Lambda$ such that $\mu\left(K \cap\left(\cup_{\lambda \in \Phi} G_{\lambda}\right)^{c}\right) \leq \epsilon$.

If there is a collection $\mathcal{H}$ of closed subsets $H$ of $E$ which verifies the following conditions:

1. every $H \in \mathcal{H}$ is $\mu$-compact,

2. $\mu(H)<\infty$ for every $H \in \mathcal{H}$, 
3. for every $B \in \mathcal{B}$ one has

$$
\mu(B)=\sup \{\mu(H): B \subset H \in \mathcal{H}\},
$$

then $\mu$ is said to be Radon measure of type $(\mathcal{H})$.

A possible choice for $\mathcal{H}$ is the collection $\mathcal{F}$ of all finite subsets of $E$. Clearly the counting measure is a Radon measure of type $(\mathcal{F})$ on any topological space. Other possibilities are: the collection $\mathcal{K}$ of all compact sets (this is the choice of Schwartz), the collection $\mathcal{K}_{m}$ of all metrizable compact sets, and the collection $\mathcal{F}_{\varphi}$ of all closed subsets with finite measure. A Radon measure of type $(\mathcal{H})$ is not assumed necessarily to be locally finite.

Let $E$ be a not necessarily Hausdorff topological space and $\mu^{*}$ an outer measure on $E$, that is a set function defined on the collection $\mathcal{P}(E)$ of all subsets of $E$ which satisfies the following conditions:

1. $0 \leq \mu^{*}(X) \leq \infty$ for $X \subset E$,

2. $\mu^{*}(\emptyset)=0$,

3. $\mu^{*}(X) \leq \mu^{*}(Y)$ if $X \subset Y, X, Y \in \mathcal{P}(E)$,

and $\mu^{*}$ is countably subadditive, i.e.

4. $\mu^{*}\left(\cup_{n} X_{n}\right) \leq \Sigma_{n} \mu^{*}\left(X_{n}\right)$ if $\left(X_{n}\right)$ is a sequence of subsets of $E$.

Rodríguez-Salinas and Jiménez Guerra say that $\mu^{*}$ is a topological outer measure if furthermore: i.e.

a) every Borel subset of $E$ is $\mu^{*}$-measurable in the sense of Carathédory,

$$
\mu^{*}(X)=\mu^{*}(X \cap B)+\mu^{*}\left(X \cap B^{c}\right) \text { for } X \in \mathcal{P}(E), B \in B,
$$

b) $\mu^{*}$ is locally finite, i.e. every point $x \in E$ has a neighborhood $V$ such that $\mu^{*}(V)<\infty$,

c) if $\left(G_{\lambda}\right)$ is an increasingly directed family of open subsets of $E$ then

$$
\mu^{*}\left(\cup_{\lambda} G_{\lambda}\right)=\sup _{\lambda} \mu^{*}\left(G_{\lambda}\right)
$$

d) for every set $X \subset E$ one has

$$
\mu^{*}(X)=\inf \left\{\mu^{*}(G): X \subset G \in \mathcal{G}\right\}
$$


where $\mathcal{G}$ denotes the collection of all open subsets of $E$.

They call the restriction $\mu$ of $\mu^{*}$ to $\mathcal{B}$ (or to the collection of all $\mu^{*}$ measurable sets) a topological measure. They also introduce the essential outer measure $\mu^{*}$ associated with $\mu^{*}$ by setting

$$
\mu^{\bullet}(X)=\sup \left\{\mu^{*}(X \cap G): G \in \mathcal{G}_{0}\right\},
$$

where $\mathcal{G}_{0}$ is the collection of those $G \in \mathcal{G}$ for which $\mu^{*}(G)<\infty$.

Let now $\mu$ be a locally finite Radon measure of type $(\mathcal{H})$, where $\mathcal{H}$ is an increasingly directed collection of closed subsets of $E$. For any $X \subset E$ the authors set

$$
\mu_{*}(X)=\sup \{\mu(H): X \supset H \in \mathcal{H}\},
$$

and for $H \in \mathcal{H}$ and $X \subset H$ they define

$$
\mu_{H}^{*}(X)=\mu(H)-\mu_{*}\left(H \cap X^{c}\right)=\inf \{\mu(B): X \subset B \in \mathcal{B}\} .
$$

Then $\mu_{H}^{*}$ is a topological outer measure on $H$ and

$$
\mu^{\bullet}(X)=\sup \left\{\mu_{H}^{*}(X \cap H): H \in \mathcal{H}\right\}
$$

is the essential outer measure associated with the topological outer measure

$$
\mu^{*}(X)=\inf \{\mu(G): X \subset G \in \mathcal{G}\} .
$$

Using these concepts and techniques Rodríguez-Salinas and Jiménez Guerra generalize the results in the book of Laurent Schwartz. Thus where Schwartz imposes the condition that the compact subsets of $E$ are metrizable, they consider Radon measures of type $\left(\mathcal{K}_{m}\right)$. They are able to define the measure induced on any subset of $E$ not just a measurable one. They prove the existence of a "concassage" for a locally $\sigma$-finite Radon measure of type $(\mathcal{H})$, furthermore a Lebesgue-Nikodým theorem, the existence of tensor products, and most importantly theorems on the existence of the projective limit of a family of measures.

Let $\mu$ be a finite Radon measure of type $(\mathcal{H})$ and $E$ a Hausdorff topological space. Consider a sequence $\left(C_{n}\right)_{n \in \mathbb{N}}$ of countable sets, a sequence $\left(p_{n}\right)_{n \in \mathbb{N}}$ of surjective maps $p_{n}: C_{n+1} \rightarrow C_{n}$, and a sequence $\left(\varphi_{n}\right)_{n \in \mathbb{N}}$ of maps $\varphi_{n}: C_{n} \rightarrow \mathcal{P}(E)$. Rodríguez-Salinas says that $E$ is a $\mu$-Suslin space [71] if the following conditions are satisfied: 
1. $\varphi_{n+1}(c) \subset \varphi_{n}\left(p_{n}(c)\right)$ for $c \in C_{n+1}, n \in \mathbb{N}$,

2. the sets

$$
E-U\left\{\varphi_{0}(c): c \in C_{0}\right\}
$$

and

$$
\varphi_{n}(c)-\cup\left\{\varphi_{n+1}(d): d \in C_{n+1}, p_{n}(d)=c\right\}, \quad c \in C_{n}, \quad n \in \mathbb{N}
$$

have $\mu$-measure zero,

3. let $\left(c_{n}\right)$ be a coherent sequence, i.e. $c_{n} \in C_{n}$ and $p_{n}\left(c_{n+1}\right)=c_{n}$; then the sets $\varphi_{n}\left(c_{n}\right)$ converge to a point $x \in E$ contained in each set $\varphi_{n}\left(c_{n}\right)$.

He says that $E$ is a $\mu$-Lusin space if furthermore:

$\left(^{*}\right)$ each $\varphi_{n}$ is injective and the sets $\varphi_{n}(c), c \in C_{n}$, are pairwise disjoint.

Each set $\varphi_{n}(c)$ is then $\mu$-measurable. The space $E$ is semi- $\mu$-Suslin if $\left(C_{n}, p_{n}, \varphi_{n}\right)$ satisfies conditions 1 . and 2. above and furthermore:

$0)$ each set $\varphi_{n}(c), c \in C_{n}, n \in \mathbb{N}$, is $\mu$-measurable,

$\left.3^{\prime}\right)$ if $\left(c_{n}\right)$ is a coherent sequence and $x \in \Pi_{n} \varphi_{n}\left(c_{n}\right)$, then $\varphi_{n}\left(c_{n}\right)$ converges to $x$.

The space $E$ is semi- $\mu$-Lusin if furthermore $\left({ }^{*}\right)$ is satisfied. We have the following implications:

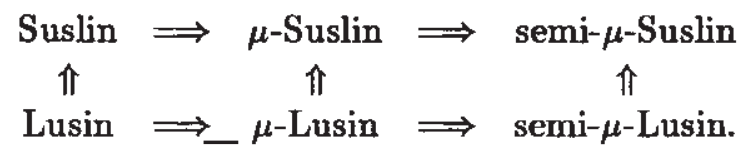

Theorem [71]. Let $E$ be a Hausdorff space and $\mu$ a positive Borel measure on $E$ such that $\mu(E)=1$. The following are equivalent:

1. $E$ is $\mu$-Suslin.

2. $\mu$ is a Radon measure of type $\left(\mathcal{K}_{m}\right)$.

3. There exists a sequence $\left(C_{n}, p_{n}, \varphi_{n}\right)$ such that each $C_{n}$ consists of compact, metrizable, pairwise disjoint subsets of $E$ and $\varphi_{n}$ is the identity. 


\section{4. $E$ is $\mu$-Lusin.}

Let $\mathcal{L}^{\infty}$ be the algebra of essentially bounded measurable functions, $L^{\infty}$ the usual quotient space modulo the functions almost everywhere equal to zero, and $\pi$ the canonical surjection $\mathcal{L}^{\infty} \rightarrow L^{\infty}$. An algebra homomorphism $\rho: \mathcal{L}^{\infty} \rightarrow \mathcal{L}^{\infty}$ is a lifting $\{14\}$ if it satisfies the following conditions:

(I) $\rho(f)$ is almost everywhere equal to $f$;

(II) if $f$ is almost everywhere equal to $g$, then $\rho(f)=\rho(g)$.

The name "lifting" is due to the fact that $\rho$ permits to define a section $\sigma: L^{\infty} \rightarrow \mathcal{L}^{\infty}$ of $\pi$ by setting $\sigma(\pi(f))=\rho(f)$. A lifting $\rho$ is almost strong if there exists a set $A \subset E$ such that $\mu^{\bullet}\left(A^{c}\right)=0$ and for all bounded continuous functions $f$ the restrictions of $f$ and of $\rho(f)$ to $A$ are equal. Don Baltasar proves [71] that if $E$ is a semi- $\mu$-Lusin space then every lifting is almost strong.

A Radon measure $\mu$ of type $(\mathcal{H})$ is strict if for every Borel set $B$ one has

$$
\mu(B)=\inf \{\mu(G): B \subset G \in \mathcal{G}\} .
$$

Let $E$ be a uniform space and $h$ a function which associates with each surrounding (= entourage) $U$ a strictly positive number $h(U)$ such that $U \subset V$ implies $h(U) \leq h(V)$ and $\lim _{U} h(U)=0$, where the limit is with respect to the decreasingly directed set of surroundings. For $x \in E$ let $U[x]$ be the neighborhood $\{y \in E:(x, y) \in U\}$ of $x$ and write $U[A]=\cup_{x \in A} U[x]$. Generalizing a result of P. Mattila $\{20\}$, don Baltasar proves [91] the following result concerning the differentiation of measures on the uniform space $E$ : If $\mu$ is a strict Radon measure of type $(\mathcal{H})$ on $E$, the set $A \subset E$ has $\sigma$-finite $\mu$-measure and $f \in L^{p}(\mu)$, then

$$
\lim _{U} \frac{1}{h(U)} \int_{A} \int_{U[x]}|f(y)-f(x)|^{p} d \mu(y) d \mu(x)=0 .
$$

Rodríguez-Salinas and Jiménez Guerra [90] prove Fubini theorems of which I quote the following: Let $X$ and $Y$ be two topological spaces, $\mu$ a Radon measure of type $\left(\mathcal{H}_{1}\right)$ on $X$, and $\nu$ a Radon measure of type ' $\left(\mathcal{H}_{2}\right)$ on $Y$. Assume that the tensor product $\mu \otimes \nu$ exists; this is for instance the case when each $\left(\mathcal{H}_{k}\right)(k=1,2)$ is increasingly directed and 
every $H \in \mathcal{H}_{k}$ is quasiregular for the induced topology. Let $f$ be a $\mu \otimes \nu$ integrable function on $X \times Y$. Then $f_{x}: y \mapsto f(x, y)$ is $\nu$-integrable for $\mu$-almost every $x \in X$ and

$$
\int_{X \times Y} f(x, y) d(\mu \otimes \nu)=\int_{X}\left(\int_{Y} f(x, y) d \nu(y)\right) d \mu(x) .
$$

2) Let $E$ be a vector space equipped with a locally convex Hausdorff topology $\tau$. Let me recall that a subset $M$ of $E$ is precompact if every ultrafilter on $M$ is a Cauchy filter. Denote by $\mathcal{M}$ the collection of all precompact subsets of $E$. On the topological dual $E^{\prime}$ of $E$ the topology $\mathcal{T}^{0}$ polar to $\mathcal{T}$ is the topology of uniform convergence on the sets $M \in \mathcal{M}$. The space $E$ is said to be polar semi-reflexive if the topological dual of $E^{\prime}$ equipped with $\mathcal{T}^{0}$ coincides as a vector space with $E$. For this it is necessary and sufficient that every precompact subset of $E$ be relatively compact $\{15\}$.

Rodríguez-Salinas constructs a theory of integration for functions $f:(\Omega, \Sigma, \mu) \rightarrow E$, where $\Sigma$ is a $\sigma$-algebra of subsets of a set $\Omega$, and $E$ is a polar semi-reflexive space. He supposes first [75] that the measure $\mu$ defined on $\Sigma$ is finite, and later [93] considers arbitrary measures. Denote by $S_{0}$ the space of $\Sigma$-measurable functions $f$ which assume only finitely many values in $E$, i.e. of the form $f=\sum_{i=1}^{n} y_{i} \chi_{A_{i}}$, where $A_{i} \in \Sigma$ and $0 \neq y_{i} \in E$. Let $S$ be the space which consists of the uniform limits of nets of functions belonging to $S_{0}$. It is easy to see that if $f \in S$, then $f(\Omega)$ is precompact in $E$. Let $\Sigma_{0}$ denote the collection of those $A \in \Sigma$ for which $\mu(A)<\infty$. Don Baltasar says that $f: \Omega \rightarrow E$ is $\mu$-measurable if for every $A \in \Sigma_{0}$ there exists a net $\left(f_{i}\right)$ of functions belonging to $S$ which converges almost uniformly to $f$ on $A$, i.e. for every $\epsilon>0$ there exists a subset $B$ of $A$ such that $\mu(B) \leq \epsilon$ and $\left(f_{i}\right)$ converges uniformly to $f$ on $A \cap B^{c}$. The function $f$ is totally $\mu$-measurable if the same net ( $\left.f_{i}\right)$ can be used for every $A \in \Sigma_{0}$. Denote by $M=M(\Sigma, \mu ; E)$ the vector space of $\mu$-measurable functions, and by $M_{t}=M_{t}(\Sigma, \mu ; E)$ that of totally $\mu$-measurable functions. Clearly $S_{0} \subset S \subset M_{t} \subset M$.

The measure space $(\Omega, \Sigma, \mu)$ is strictly localizable if there is a partitioning $\Omega=\cup_{\lambda \in \Lambda} \Omega_{\lambda}$ into pairwise disjoint sets $\Omega_{\lambda} \in \Sigma_{0}$ such that for every set $A \in \Sigma_{0}$ there exists a countable subset $\Lambda_{0}$ of the index set $\Lambda$ for which $\mu\left(A \cap\left(\cup_{\lambda \in \Lambda_{0}} \Omega_{\lambda}\right)^{c}\right)=0$. Rodríguez-Salinas proves that if $(\Omega, \Sigma, \mu)$ is strictly localizable, then $M_{t}=M$. He is introduces two further concepts of measurability: 
The function $f: \Omega \rightarrow E$ is $\bar{\mu}$-measurable if for every $A \in \Sigma_{0}$ there exists a net of $\mu$-measurable functions which converges to $f$ uniformly on $A$. The function $f$ is totally $\bar{\mu}$-measurable if there exists a net of totally $\mu$-measurable functions which converges to $f$, uniformly on every set $A \in \Sigma_{0}$.

If $f$ is $\bar{\mu}$-measurable then $f$ is essentially $\omega$-precompact, i.e. for every $A \in \Sigma_{0}$ and every neighborhood $V$ of 0 in $E$ there exists a subset $Z$ of $A$ with $\mu(Z)=0$ and a countable subset $N$ of $E$ such that $f\left(A \cap Z^{c}\right) \subset$ $N+V$.

For $f=\sum_{i=1}^{n} y_{i} \chi_{A_{i}} \in S_{0}$ the integral on $A \in \Sigma$ is defined by

$$
\int_{A} f d \mu=\sum_{i=1}^{n} y_{i} \mu\left(A \cap \dot{A}_{i}\right)
$$

provided that $\sum_{i=1}^{n} \mu\left(A \cap A_{i}\right)<\infty$, and $f$ is said to be integrable if it is integrable on $\Omega$, i.e. if $\sum_{i=1}^{n} \mu\left(A_{i}\right)<\infty$. For $f \in S$ and $A \in \Sigma_{0}$ one defines

$$
\int_{A} f d \mu=\lim _{i} \int_{A} f_{i} \cdot d \mu
$$

where $\left(f_{i}\right)$ is a net of functions in $S_{0}$ which converges uniformly to $f$. The limit exists and is independent of the choice of the net. One has

$$
\int_{A} f d \mu \in \mu(A) \cdot f(\Omega)^{00}
$$

since $f(\Omega)$ is precompact and $E$ is polar semi-reflexive the bipolar $f(\Omega)^{00}$ belongs to $E$ and so does the integral.

A function $f \in S$ is integrable if the following conditions are satisfied:

1) There exists a subset $\Omega_{0} \in \Sigma$ of $\Omega$ such that $f$ vanishes outside $\Omega_{0}$ and $\Omega_{0}$ is strictly localizable, i.e. it has a partitioning $\left(\Omega_{\lambda}\right)_{\lambda \in \Lambda}$ into pairwise disjoint sets $\Omega_{\lambda} \in \Sigma_{0}$ with the above described properties.

2) For every $A \in \Sigma$ the family $\left(\int_{A \cap \Omega_{\lambda}} f d \mu\right)_{\lambda \in \Lambda}$ is summable in $E$.

The integral of $f$ on $A \in \Sigma$ is then defined by

$$
\int_{A} f d \mu=\sum_{\lambda \in \Lambda} \int_{A \cap \Omega_{\lambda}} f d \mu
$$

It is independent of the choice of $\Omega_{0}$ and of the $\Omega_{\lambda}$. 
At this point Rodríguez-Salinas supposes that the measure $\mu$ is essential, i.e. that for every $A \in \Sigma$ one has

$$
\mu(A)=\sup \left\{\mu(A \cap K): K \in \Sigma_{0}\right\} .
$$

The function $f: \Omega \rightarrow E$ is said to be $\mu$-integrable (resp. totally $\mu$-integrable) if:

1) There exists a strictly localizable subset $\Omega_{0} \in \Sigma$ of $\Omega$ such that $f(t)=0$ for $t \in \Omega_{0}^{c}$.

2) $f$ is $\mu$-measurable (resp. totally $\mu$-measurable).

3) $f$ is Pettis-integrable, i.e. for every $x^{\prime} \in E^{\prime}$ the numerical function $t \mapsto\left\langle f(t), x^{\prime}\right\rangle$ is $\mu$-integrable and for all $A \in \Sigma$ there exists $m_{f}(A) \in E$ such that

$$
\int_{A}\left\langle f(t), x^{\prime}\right\rangle d \mu(t)=\left\langle m_{f}(A), x^{\prime}\right\rangle
$$

Then the integral of $f$ on $A$ is $m_{f}(A)$.

Rodriguez-Salinas introduces furthermore the concept of a $\bar{\mu}$-integrable function $f: \Omega \rightarrow E$ by means of the following conditions:

0) $f$ is $\bar{\mu}$-measurable.

1) There exists a strictly localizable subset $\Omega_{0} \in \Sigma$ of $\Omega$ such that $f(t)=0$ for $t \in \Omega_{0}^{c}$.

2) For every $A \in \Sigma_{0}$ the function $f$ is the uniform limit on $A$ of a net $\left(f_{i}\right)$ of $\mu$-integrable functions such that $\lim _{i} \int_{A} f_{i} d \mu$ exists in $E$. Then the limit does not depend on the choice of the approximating net and one sets

$$
\int_{A} f d \mu=\lim _{i} \int_{A} f_{i} d \mu
$$

3) If $\left(\Omega_{\lambda}\right)_{\lambda \in \Lambda}$ is the partition of $\Omega_{0}$ which figures in the definition of the strict localizability of $\Omega_{0}$, then for all $A \in \Sigma$ the family $\left(\int_{A \cap \Omega_{\lambda}} f d \mu\right)_{\lambda \in \Lambda}$ is summable in $E$.

In this case the $\bar{\mu}$-integral of $f$ on $A \in \Sigma$ is, of course, defined by

$$
\int_{A} f d \mu=\sum_{\lambda \in \Lambda} \int_{A \cap \Omega_{\lambda}} f d \mu .
$$


Every $\bar{\mu}$-integrable function is Pettis integrable but there exists $\bar{\mu}-$ integrable functions that are not $\mu$-measurable.

Let $\mathcal{P}$ be the collection of all continuous seminorms on $E$. Two functions $f$ and $g$ from $\Omega$ into $E$ are $\mu$-equivalent (in symbol: $f \cong g$ ) if

$$
\mu(\{t \in \Omega: p(f(t)-g(t)) \neq 0\})=0
$$

for all $p \in \mathcal{P}$. The essential range of $f: \Omega \rightarrow E$ on $A \in \Sigma$ is defined by

$$
\operatorname{er}_{A}(f)=\{x \in E: \mu(\{t \in A: p(f(t)-x) \leq 1\}) \neq 0, \quad \forall p \in \mathcal{P}\} .
$$

Among the several lifting theorems Rodriguez-Salinas proves in Part IV of [93] let me quote the following:

Let $(\Omega, \Sigma, \mu)$ be a strictly localizable measure space, $\rho: S \rightarrow S$ a lifting and $f$ a $\mu$-measurable function. Then there exists a $\mu$-measurable function $g$ such that $f \cong g, \rho(g)=g$ and for every $A \in \Sigma_{0}$ with $\mu(A)>0$ one has $g(t) \in \operatorname{er}_{A}(f)=\operatorname{er}_{A}(g)$ for almost every $t \in A$. Furthermore $g(A) \subset \operatorname{er}_{A}(g) \cup\{0\}$ if $\rho(A) \supset A$.

Between 1980 and 1984 Rodríguez-Salinas wrote a number of articles on the Radon-Nikodỳm theorem for vector-valued measures, all published in the Revista of the Royal Academy of Madrid [77], [78], [94], [96], [98], [99]. I can only give a smali sample of his results. Let $(\Omega, \Sigma, \mu)$ be a complete measure space with $\mu(\Omega)<\infty$. Let $E$ be a locally convex Hausdorff space and $m: \Sigma \rightarrow E$ a vector measure. Denote by $\Sigma^{+}$the collection of those $A \in \Sigma$ for which $\mu(A)>0$. Set

$$
A_{\Omega}(m)=\left\{\frac{m(A)}{\mu(A)}: A \in \Sigma^{+}\right\}
$$

The measure $m$ is controlled by $\mu$ if $m$ is $\mu$-continuous and $A_{\Omega}(m)$ is bounded. The space $E$ has the Radon-Nikodỳm property if for every $(\Omega, \Sigma, \mu)$ and every $m: \Sigma \rightarrow E$ controlled by $\mu$ there exists a $\bar{\mu}$-integrable function $f: \Omega \rightarrow E^{\prime \prime}$ such that $m(A)=\int_{A} f(t) d \mu(t)$ for each $A \in \Sigma$.

The set $A \subset E$ is dentable if for every neighborhood $V$ of 0 in $E$ there exists an $x \in A$ such that $x$ does not belong to the closed convex hull of $A \cap(x+V)^{c}$. The set $A \subset E$ is $\sigma$-dentable if for every neighborhood $V$ of 0 in $E$ there exists $x \in A$ such that $x \notin \sigma\left(A \cap(x+V)^{c}\right)$, where $\sigma(X)=\left\{\sum_{n \in \mathbb{N}} \lambda_{n} x_{n}: x_{n} \in X, \lambda_{n}>0, \sum_{n \in \mathbb{N}} \lambda_{n}=1\right\}$. 
Theorem [96]. Let $E$ be a locally convex Hausdorff space and assume that its dual $E^{\prime}$ equipped with the strong topology $\beta\left(E^{\prime}, E\right)$ is quasicomplete (this is the case e.g. when $E$ is barrelled, and it implies that $E^{\prime}$ is polar semi-reflexive). Let us furthermore assume that for every countable bounded subset $B$ of $E$ the closed subspace $F$ generated by $B$ is infrabarrelled. Then the following ane equivalent:

i) $E^{\prime}$ has the Radon-Nikodỳm property.

ii) Every countable bounded non-empty subset of $E^{\prime}$ is dentable.

iii) Every bounded non-empty subset of $E^{\prime}$ is $\sigma$-dentable.

iv) Every equicontinuous non-empty subset $B$ of $E^{\prime}$ has the property that for every neighborhood $V$ of 0 in $E^{\prime}$ there is an $x^{\prime} \in B$ such that $x^{\prime}$ does not belong to the convex hull of $B \cap\left(x^{\prime}+V\right)^{c}$.

v) For each of the above mentioned subspaces $F$ every bounded subset of the strong dual $F^{\prime}$ is w-precompact.

The same conclusion holds if $E$ is supposed to be infrabarrelled [98] (without any conditions on $E^{\prime}$ or $F$ ).

Theorem [94]. Let $E$ be a quasi-complete locally convex Hausdorff space. The following are equivalent:

i) E has the Radon-Nikodỳm property.

ii) Every martingale $\left(f_{i}, \Sigma_{i}\right)$ with values in the space $E$, such that the $f_{i}$ are uniformly bounded, is a Cauchy net in the space $\mathcal{L}^{1}(\Omega, \Sigma, \mu ; E)$ of $\mu$-integrable functions.

iii) Every martingale $\left(f_{n}, \Sigma_{n}\right)_{n \in \mathbb{N}}$ with values in $E$, such that the $f_{n}$ are uniformly bounded, is a Cauchy sequence in the space $\overline{\mathcal{L}}^{1}(\Omega, \Sigma, \mu ; E)$ of $\bar{\mu}$-integrable functions.

iv) Every countable bounded non-empty subset $E$ is $\sigma$-dentable.

v) Every bounded non-empty subset $E$ is $\sigma$-dentable. 
vi) For every countable bounded non-empty subset $B$ of $E$ and every neighborhood $V$ of 0 in $E$ there exists $x \in B$ such that $x$ does not belong to the convex hull of $B \cap(x+V)^{c}$.

vii) For every bounded non-empty subset $B$ of $E$ and every neighborhood $V$ of 0 in $E$ there exists $x \in B$ such that $x$ does not belong to the convex hull of $B \cap(x+V)^{c}$.

viii) For every bounded non-empty subset $B$ of $E$ and every balanced convex closed neighborhood $V$ of 0 in $E$ the set $\pi_{V}(B)$ is dentable (or, equivalently, $\sigma$-dentable), where $\pi_{V}$ denotes the canonical surjection from $E$ onto the quotient space $E / \cap_{\lambda>0} \lambda U$.

Rodríguez-Salinas has written about twenty more works on the theory of integration. Ten of these papers were written in collaboration with José L. DeMaria. Some of their results are summarized in the expository lecture [119] which introduces such concepts as Spanish $\sigma$ algebras and Spanish sets. Besides the papers referred to above, don Baltasar wrote other papers on integration with Pedro Jiménez Guerra. One of the papers written jointly with Fernando Bombal Gordón [103] is related to the topics discussed above. Let me observe that Bombal developed a parallel theory of integration for functions with values in a vector space $\{3\},\{4\}$, which, however, is not assumed to be topological but bornological.

Don Baltasar is still very active and his mathematical output has not diminished. I wish him many more years of healthy, happy mathematical activity.

\section{References}

\{1\} Arias de Reyna Martínez, Juan: $\ell_{0}^{\circ}(\Sigma)$ no es totalmente tonelado. Rev. Real Acad. Cienc. Exact. Fís Natur. Madrid 74 (1985), 77-78.

$\{2\}$ Bang, Thøger: Om quasi-analytiske funktioner. Nyt Nordisk Forlag-Amold Busck, Copenhagen, 1946.

\{3\} Bombal Gordón, Fernando: Medida e integración en espacios bornológicos. Rev. Real Acad. Cienc. Exact. Fís .Natur. Madrid 75 (1981), 115-137. 
\{4\} Bombal Gordón, Fernando: El teorema de Radon-Nikodym en espacios bornológicos. Rev. Real Acad. Cienc. Exact. Fís. Natur. Madrid 75 (1981), 139-154.

\{5\} Bombal Gordón, Fernando and Cembranos Diaz, Pilar: The Dieudonné property on $C(K, E)$. Trans. Amer. Math. Soc. 285 (1984), 649-656.

\{6\} Bourbaki, Nicolas: Éléments de mathématique, Intégration, Chaps. I-IV, V, VI. Hermann, Paris, 1965, 1967, 1959.

\{7\} Butzer, Paul L., Gieseler, S., Kaufmann, F., Nessel, R.J. and Stark, A.L.: Eduard Helly (1884-1943)-Eine nachträgliche Würdigung. Jahresber. Deutsch. Math.-Verein. 82 (1980), 128-151.

\{8\} Diestel, Joseph and Uhl, John Jerry: Vector measures. Mathematical Surveys No. 15, American Mathematical Society, Providence, R.I., 1977.

\{9\} Ferrando, Juan Carlos and López Pellicer, Manuel: Strong barrelledness properties in $\ell_{0}^{\infty}(X, \mathcal{A})$ and bounded finite additive measures. Math. Ann. 287 (1990), 727-736.

\{10\} Ferrando, Juan Carlos and López Pellicer, Manuel: On exhaustive vector measures. Rev. Colombiana Mat. 28 (1994), 13-21.

\{11\} Ferrando, Juan Carlos., López Pellicer, Manuel and Sánchez Ruíz, L.M.: Metrizable barrelled spaces. Pitman Research Notes in Mathematics Series No. 332, Longman, Harlow, Essex, 1995.

\{12\} Grothendieck, Alexandre: Sur les applications linéaires faiblement compactes d'espaces du type $C(K)$. Canad. J. Math. 5 (1953), 129-173.

\{13\} Halmos, Paul R.: Measure theory. D. van Nostrand, Princeton, N.J., 1950. 
\{14\} Ionescu Tulcea, Alexandra and Ionescu Tulcea, Cassio: Topics in the theory of lifting. Ergebnisse der Mathematik und ihrer Grenzgebiete Bd. 48, SpringerVerlag, Berlin-Heidelberg-New York, 1969.

\{15\} Köthe, Gottfried: Topological vector spaces I, II. SpringerVerlag, Berlin-Heidelberg-New York, 1969, 1979.

\{16\} Kunzinger, Michael: Barrelledness, Baire-like- and (LF)spaces. Pitman Research Notes in Mathematics Series No. 298, Longman, Harlow; Essex, 1993.

$\{17\}$ López Pellicer, Manuel: Clases de tonelacion y medidas acotadas. Rev: Real Acad. Cienc. Exact. Fís. Natur. Madrid 84 (1990), 541-567.

\{18\} López Pellicer, Manuel: Webs and bounded finitely additive measures. J. Math. Anal. Appl.

\{19\} Mandelbrojt, Szolem: Séries adhérentes, régularisation des suites, applications. Gauthier-Villars, Paris, 1952.

\{20\} Mattila, Pertti: Differentiation of measures on uniform spaces. In "Measure Theory, Oberwolfach 1979", pp. 261-283. Lecture Notes in Mathematics No. 794, Springer-Verlag, BerlinHeidelberg-New York, 1980.

\{21\} Robertson, Wendy J., Tweddle, I., Yeomans, F.E.: On the stability of barrelled topologies III. Bull. Austral. Math. Soc. 22 (1980), 99-112.

\{22\} Schwartz, Laurent: Radon measures on arbitrary topological spaces and cylindrical measures. Tata Institute of Fundamental Research, Studies in Mathematics No. 6, Oxford University Press, London, 1973.

\{23\} Valdivia Ureña, Manuel: On certain barrelled normed spaces. Ann. Inst. Fourier (Grenoble) 29 (1979), fasc. 3, 39-56.

\{24\} Valdivia Ureña, Manuel: On suprabarrelled spaces. In "Functional Analysis, Holomorphy and Approximation Theory, Rio 
de Janeiro 1978", pp. 572-580. Lecture Notes in Mathematics No. 843, Springer-Verlag, Berlin-Heidelberg-New York, 1981.

\{25\} Valdivia Ureña, Manuel and Pérez Carreras, Pedro: On totally barrelled spaces. Math. Z. 178 (1981), 263-269. 


\section{The Mathematical Works of Baltasar Rodríguez- Salinas}

I shall abbreviate the name of the "Revista de la Real Academia de Ciencias Exactas; Físicas y Naturales de Madrid" by "Rev. Acad. Ci". Otherwise I use the abbreviations of Mathematical Reviews.

[1] Determinante que da $f(x+y)$ en función de $f(x) y$ de $f(y)$. (A determinant which yields $f(x+y)$ in terms of $f(x)$ and of $f(y)$.) Euclides 2 (1942), 159-162.

[2] Resolución de la ecuación funcional: (Solution of the functional equation:)

$$
F(x)=-\frac{F\left[x+2 F(x) F^{\prime}(x)\left(1+{\overline{F^{\prime}(x)}}^{2}\right)\right]}{1+2{\overline{F^{\prime}}(x)}^{2}} .
$$

Euclides 2 (1942), 349-351.

[3] Una demostración sobre la integral de Dirichlet. (A proof on Dirichlet's integral.) Euclides 2 (1942), 425.

[4] Modo de sumar algunas expresiones. (A method to sum certain expressions.) Euclides 3 (i943), 343-348.

[5] La inversion en el orden de la derivación. (The inversion of the order of differentiation.) Rev. Acad. Ci. 42 (1948), 37-70.

[6] Sobre la teoria de la medida. (On the theory of measure.) Rev. Acad. Ci. 42 (1948), 465-491.

[7] Sobre una función meromorfa y sus aplicaciones a la suma de series. (On a meromorphic function and its applications to the summation of series.) Gaceta Mat. 3 (1951), 6-17.

[8] Sobre la determinación de una función analítica conocida su parte real. (On the determination of an analytic function when its real part is known.) Gaceta Mat. 4 (1952), 44-46.

[9] Sobre el comportamiento asintótico de la aplicación reiterada de una sucesión de funciones. (On the asymptotic behavior of the 
iterated application of a sequence of functions.) Gaceta Mat. 4 (1952), 81-90.

[10] Sobre la región de valores de una función lisa. (On the region of values of a schlicht function.) Rev. Mat. Hispano-Americana (4) 12 (1952), 223-228.

[11] Sobre una generalización de las fórmulas de Taylor, Darboux y Euler-Maclaurin. (On a generalization of the formulas of Taylor, Darboux and Euler-Maclaurin.) Rev. Mat. Hispano-Americana (4) 12 (1952), 281-289.

[12] Sobre varias formas de proceder en la determinación de periodos de las mareas y predicción de las mismas en un cierto lugar. (On various procedures for the determination of the periods of tides and their prediction at a particular place.) Rev. Acad. Ci 46 (1952), 441-457.

[13] Transformadas de Laplace de algunas funciones integrales. (Laplace transforms of some entire functions.) Gaceta Mat. 5 (1953), 157-158.

[14] Sobre ciertos desarrollos asintóticos de integrales de Laplace curvilíneas. (On certain asymptotic expansions of curvilinear Laplace integrals.) Rev. Mat. Hispano-Americana (4) 13 (1953), 120-127.

[15] (with Ricardo San Juan) Exposición sobre algunos teoremas conocidos y otros nuevos sobre convergencia ordinaria y uniforme de la integral de Fourier. (Expository account of theorems, some known, some new, on the ordinary and the uniform convergence of the Fourier integral.) Rev. Acad. Ci. 47 (1953), 495-510.

[16] Complemento a un teorema de Ahlfors-Heins sobre funciones subarmónicas. (Complement to a theorem of Ahlfors-Heins on subharmonic functions.) Rev. Acad. Cienc. Zaragoza (2) 9 (1954), $119-125$.

[17] Funciones con momentos nulos. (Functions with zero moments.) Rev. Acad. Ci. 49 (1955), 331-368. 
[18] Los problemas de unicidad en la teoria de series asintóticas. Expresión de funciones semi-analíticas mediante algoritmos de Borel y Stieltjes. (Uniqueness problems in the theory of asymptotic series. Expression of semi-analytic functions by means of algorithms of Borel and Stieltjes.) Rev. Acad. Ci 50 (1956), 191227.

[19] Sobre la ecuación diferencial: (On the differential equation:)

$$
\frac{d^{2} y}{\overline{d x^{2}}}+\left[\frac{a_{0}+a_{1} \cos 2 x}{b_{0}+b_{1} \cos 2 x}-\frac{m(m-1)}{\cos ^{2} x}-\frac{n(n-1)}{\underline{\operatorname{sen}^{2} x}}\right] y=0 \text {. }
$$

Rev. Mat. Hispano-Americana(4) 15 (1995), 31-47, 121-135, 182-208; 16 (1956), 49-71, 122-150, 229-263.

[20] Sobre una ecuación diferencial. ( $\mathrm{On}$ a differential equation.) Memorias de Matemática del Instituto "Jorge Juan", No. 18. Consejo Superior de Investigaciones Cientificas, Madrid 1956.i + $145 \mathrm{pp}$.

[21] Moments de fonctions analytiques et problème de Watson. (Moments of analytic functions and Watson's problem.) J. Math. Pures Appl.(9) 35 (1956), 359-382.

[22] Ceros de las funciones de una clase no cuasi-analítica en $R^{n}$. Prolongación no cuasi-analítica. (Zeros of the functions from a non quasi-analytic class in $\boldsymbol{R}^{n}$. Non quasi-analytic continuation.) Collect. Math. 9 (1957), 65-77.

[23] Una desigualdad entre las cotas de las derivadas de una función analítica en un angulo. (An inequality between the bounds of the derivatives of an analytic function in an angle.) Las Ciencias, Madrid 23 (1958), 533-539.

[24] Disuguaglianze tra limiti e coefficienti dello sviluppo asintotico di una funzione in un angulo. (Inequalities between bounds and coefficients of the asymptotic expansion of a function in an angle.) Ann. Mat. Pura Appl (4) 48 (1959), 147-159. 
[25] Sulla stabilità delle soluzioni per l'equazione differenziale del secondo ordine a coefficienti periodici. (On the stability of the solutions of the second order differential equation with periodic coefficients.) Rend. Circ. Mat. Palermo (2) 8 (1959), 206-224.

[26] Aproximación uniforme de una función continua por un conjunto convero de funciones continuas. (Uniform approximation of a continuous function by a convex set of continuous funcţions.) Collect. Math. 11 (1959), 175-202.

[27] Equivalenza di classi di funzioni con sviluppo asintotico in un angolo. Funzione caratteristiche. (Equivalence of classes of functions with an asymptotic expansion in an angle. Characteristic functions.) Boll. Un. Mat. Ital. (3) 14 (1959), 525-531.

[28] Sobre la estabilidad de la ecuación funcional (On the stability of the functional equation) $f(x+y)=f(x)+f(y)$. Rev. Acad. Cienc. Zaragoza (2) 14 (1959), 5-7.

[29] Variación de las raices características de una ecuación diferencial de segundo orden con coeficientes periódicos. (Variation of the characteristic roots of a second order differential equation with periodic coefficients.) Ann. Mat. Pura Appl. (4) 52 (1960), 107161.

[30] Solución de un problema de Bang de la teoría de clases cuasianaliticas. (Solution of a problem of Bang in the theory of quasianalytic classes.) Las Ciencias, Madrid 25 (1960), 257-261.

[31] Sobre el cambio de variable en las integrales múltiples. (On the change of variables in multiple integrals.) Collect. Math. 12 (1960), 139-153.

[32] Una fórmula asintótica para algunas transformadas de Laplace. (An asymptotic formula for some Laplace transforms.) Rev. Acad. Ci. 54 (1960), 177-187.

[33] Un desarrollo asintótico de ciertas transformadas de Laplace. (An asymptotic expansion for some Laplace transforms.) Rev. Acad. Ci. 54 (1960), 167-176. 
[34] Funciones que verifican en cada punto de un intervalo a una ecuación diferencial variable con el punto. (Functions which satisfy at each point of an interval a differential equation which varies with the point.) Rev. Acad. Ci. 54 (1960), 301-311.

[35] Estabilidad y ceros de las soluciones de una ecuación diferencial de segundo orden con coeficientes periodicos. (Stability and zeros of the solutions of a second order differential equation with periodic coefficients.) Rev. Acad. Cienc. Zaragoza (2) 15 (1960), 5-9.

[36] Solución del problema de equivalencia de clases de funciones de desarrollo asintótico. (Solution of the problem of equivalence of classes of functions with asymptotic expansion.) Rev. Acad. Cienc. Zaragoza (2) 16 (1961), 47-51.

[37] Existencia de puntos de Weierstrass de funciones reales. Máximos y minimos en espacios casi-numerablemente compactos. (The existence of Weierstrass points of real functions. Maxima and minima in quasi-countably compact spaces.) Rev. Acad. Clienc. Zaragoza (2) 16(1961), 5-10.

[38] Conjunto de valores de un coeficiente diferencial. (The set of values of a derivative.) Collect. Math. 13 (1961), 3-13.

[39] Crecimiento de una función analítica en un ángulo. (Growth of an analytic function in an angle.) Collect. Math. 13 (1961), 197217.

[40] Existencia de máximo de funciones reales continuas en espacios casi numerablemente compactos. (The existence of a maximum of real continuous functions in almost countably-compact spaces.) Ann. Mat. Pura Appl. (4) 55 (1961), 375-413.

[41] Generalización sobre módulos del teorema de Hahn-Banach y sus aplicaciones. (A generalization to modules of the Hahn-Banach theorem and its applications.) Collect. Math. 14 (1962), 105-151.

[42] Sobre el último teorema de Fermat y la ecuación: (On Fermat's 
last theorem and the equation:)

$$
\frac{\partial^{n} u}{\partial x^{n}}+\frac{\partial^{n} u}{\partial y^{n}}=\frac{\partial^{n} u}{\partial z^{n}} .
$$

Rev. Faculdade de Ciências Lisboa (2) A9 (1962), 35-43.

[43] Una generalización de los teoremas de von Staudt y Darboux. (A generalization of theorems of von Staudt and Darboux.) Rev. Faculdade de Ciências Lisboa (2) A9 (1962), 77-86.

[44] Clases de funciones analiticas. Clases semi-analiticas y cuasianalíticas. (Classes of analytic functions. Semi-analytic and quasi-analy tic classes.) Rev. Acad. Cienc. Zaragoza (2) 17 (1962), 5-75; also published as Publicaciones del Seminario Matemático García de Galdeano, No. 6. Facultad de Ciencias de Zaragoza, Consejo Superior de Investigaciones Cientificas, 1963, 77 pp.

[45] Crecimiento de una función analítica en una banda de anchura no constante. (Growth of an analytic function in a strip of nonconstant width.) Proceedings of the third annual meeting of Spanish mathematicians. Collect. Math. 15 (1963), 5-22.

[46] Una clase de grupoides: tribus. Inmersión de una tribu. (A class of groupoids: tribes. Imbedding of a tribe.) Proceedings of the third annual meeting of Spanish mathematicians. Collect. Math. 15 (1963), 153-167.

[47] El problema de la extensión. (The extension problem.) Ann. Mat. Pura Appl. (4) 64 (1964), 133-189.

[48] Sobre la prolongación de funciones lineales. (On the extension of linear functionals.) Proceedings of the fourth annual meeting of Spanish mathematicians. Collect. Math. 16 (1964), 67-78.

[49] Sobre la teoría de la medida y sus fundamentos. (On the theory of measure and its foundations.) Inaugural lecture, Academia de Ciencias Exactas, Físico-Quimicas y Naturales de Zaragoza. May $2,1965$. 
[50] El método de iteración en la teoría de puntos fijos. (The iteration method in the theory of fixed points.) Proceedings of the fifth annual meeting of Spanish mathematicians. Rev. Mat. HispanoAmericana (4) 25 (1965), 174-185.

[51] Sobre la ecuación funcional $f(x+y)=f(x) f(y)$ y las funciones $a^{x}$ y $\log _{a} x$. (On the functional equation $f(x+y)=f(x) f(y)$ and the functions $a^{x}$ and $\log _{a} x$.) Proceedings of the fifth annual meeting of Spanish mathematicians. Rev. Mat. Hispano-Americana (4) 25 (1965), 112-129.

[52] Sur la décomposition d'ensembles en parties respectivement équivalentes. Quantité et mesure. (On the decomposition of sets into respectively equivalent subsets. Quantity and measure.) Comptes Rendus de la $\mathrm{III}^{e}$ Réunion du Groupement des Mathématiciens d'Expression Latine, Namur, 20-23 septembre 1965, 139-152. Centre Belge de Recherches Mathématiques, Librairie Universitaire, Louvain, 1966.

[53] Sobre un teorema del punto fijo. (On a fixed point theorem.) Rev. Mat. Hispano-Americana (4) 26 (1966), 148-150.

[54] Construcción de medidas de Borel sobre los espacios regulares. (The construction of Borel measures on regular spaces.) Actas de la Quinta Reunión Anual de Matemáticos Españoles, 34-41, Madrid, 1966.

[55] (with José Garay de Pablo) Sobre los cuerpos no conmutativos de rango cuatro respecto de su centro. (On non-commutative fields of rank four over their center.) Actas de la Quinta Reunión Anual de Matemáticos Españoles, 137-152, Madrid 1966.

[56] Panorama de la teoria de la medida. (Panorama of measure theory.) Lecture delivered at the cycle organized by the Academy of Sciences of the Colegio Mayor Universitario LaSalle of Zaragoza, February 15, 1967. 9 pp.

157] Sobre el paso al limite bajo el signo integral. (On passing to the limit under the integral sign.) Rev. Acad. Ci. 61 (1967), 471-499. 
[58] Sobre las medidas invariantes en un grupo topológico. (On invariant measures in a topological group.) Collect. Math. 18 (196667), 207-223.

[59] Puntos fijos en conjuntos ordenados. (Fixed points in ordered sets.) Publicaciones del Seminario Matemático "García de Galdeano", No. 10. Zaragoza (1969), 163-172.

[60] El problema de la medida. (The problem of measure)) I. Extensión de medidas en semigrupos. (The extension of measures in semigroups.) Rev. Mat. Hispano-Americana (4) 30 (1970), 141171; II. Funciones aditivas sobre un semigrupo ondenado. (Addtive functions on an ordered semigroup.) ibid. 30 (1970), 219-250; III. Teoría de la medida exterior en un semigrupo de Lebesgue. (The theory of outer measure in a Lebesgue semigroup.) ibid. 31 (1971), 46-85; IV. Extensión de funciones biaditivas. (Extension of bi-additive functions.) ibid. 31 (1971), 155-159; V. Extensión de A-homomorfismos. (Extension of A-homomorphisms.) ibid. 31 (1971), 160-180; VI. Módulos normados injectivos. (Injective normed modules.) ibid. 31 (1971), 223-238; VII. Medidas invariantes sobre A-módulos normados injectivos. (Invariant measures on injective normed A-modules.) ibid. 31 (1971), 253-306; also published as Memorias de Matemática del Instituto "Jorge Juan", No. 26. Consejo Superior de Investigaciones Cientificas, Madrid 1972. 207 pp.

[61] Algunos problemas y teoremas de extensión de aplicaciones lineales. (Some extension problems and theorems concerning linear maps.) Rev. Acad. Ci. 65 (1971), 677-704.

[62] Teoría de la medida sobre los espacios topológicos no localmente compactos. (The theory of measure on non locally compact topological spaces.) Rev. Mat. Hispano-Americana (4) 33 (1973), 178192, 257-274.

[63] (with Fernando Bombal) The Tychonoff product theorem for compact Hausdorff spaces does not imply the axiom of choice: a new proof. Equivalent propositions. Collect. Math. 24 (1973), 219230. 
[64] (with L. Bou Garcia) A theorem of Hahn-Banach type for arbitrary vector spaces. Boll. Un. Mat. Ital. (4) 10 (1974), 390-393.

[65] (with Fernando Bombal) Representación de inf-semirretículos en las partes de un espacio topológico. (Representation of infsemilattices in the subsets of a topological space.) Collect. Math. 26 (1975), 67-95.

[66] Los axiomas de la teoría de conjuntos. (The axioms of set theory.) Volume in honor of D. Manuel Lora-Tamayo, 41-50. Real Academia de Ciencias, Madrid 1975.

[67] Algunos resultados sobre aproximación de funciones continuas vectoriales. (Some results on the approximation of vector-valued continuous functions.) Boll. Un. Mat. Ital. (4) 11 (1975), 142147.

[68] (with Pedro Jiménez Guerra) Espacios de Radon de tipo $(\mathcal{H})$.

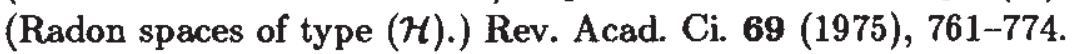

[69] Medidas en espacios topológicos. (Measures in topological spaces.) Inaugural lecture, Real Academia de Ciencias Exactas, Físicas y Naturales, Madrid, May 19, 1976.

[70] Las medidas atómicas y la $\theta$-propiedad de Darboux. (The atomic measures and Darboux's $\theta$-property.) Estudos de Álgebra, Geometria e Análise, 209-219. Academia das Ciências, Lisboa 1978.

[71] $\mu$-espacios de Suslin y Lusin. Propiedad del "lifting" fuerte. ( $\mu$ spaces of Suslin and Lusin. Strong lifting property.) Rev. Acad. Ci. 72 (1978), 541-557.

[72] Medidas de Radon de tipo $(\mathcal{H})$ y medidas con "lifting". (Radon measures of type $(\mathcal{H})$ and measures with lifting.) Rev. Acad. Ci. 72 (1978), 605-610.

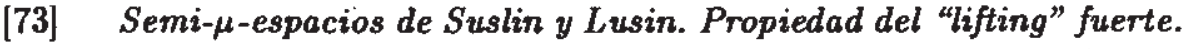
(Semi- $\mu$-spaces of Suslin and Lusin. Strong lifting property.) Rev. Acad. Ci. 73 (1979), 33-40. 
[74] Convergencia en casi todo punto de operadores definidos sobre funciones vectoriales. (The convergence at almost every point of operators defined on vector-valued functions.) Rev. Acad. Ci. 73 (1979), 157-168.

[75] Integración de funciones con valores en un espacio localmente convexo. (Integration of functions with values in a locally convex space.) Rev. Acad. Ci. 73 (1979), 361-387.

[76] (with Pedro Jiménez Guerra) Medidas de Radon de tipo $(\mathcal{H})$ en espacios topológicos arbitrarios. (Radon measures of type $(\mathcal{H})$ on arbitrary topological spaces.) Mem. Real Acad. Cienc. Exact. Fís. Natur. Madrid, Serie de Ciencias Exactas, Tomo X, 1979. 190 pp.

[77] El teorema de Radon-Nikodym para las medidas con valores en un espacio localmente convexo. (The Radon-Nikodym theorem for measures with values in a locally convex space.) Rev. Acad. Ci. 74 (1980), 41-64.

[78] La propiedad de Radon-Nikodym, $\sigma$-dentabilidad y martingalas en espacios localmente convexos. (The Radon-Nikodym property, $\sigma$ dentability and martingales in locally convex spaces.) Rev. Acad. Ci. 74 (1980), 65-89.

[79] Sobre el teorema de Dieudonné-Grothendieck. (On the Dieudonné-Grothendieck theorem.) Rev. Acad. Ci. 74 (1980), 169-171.

[80] Sobre el teorema de la gráfica cerrada. Aplicaciones lineales subcontinuas. (On the closed graph theorem. Subcontinuous linear maps.) Rev.Acad. Ci. 74 (1980), 811-825.

[81] Sobre la clase del espacio tonelado $\ell_{0}^{\infty}(\Sigma)$. (On the class of the barrelled space $\ell_{0}^{\infty \circ}(\Sigma)$.) Rev. Acad. Ci. 74 (1980), 827-829.

[82] Sobre el teorema de Dieudonné-Grothendieck. Condiciones necesarias y suficientes. (On the Dieudonne-Grothendieck theorem. Necessary and sufficient conditions.) Rev. Acad. Ci. 74 (1980), 831-834. 
[83] Sobre el teorema de la aplicación abierta. Teorema de Seever. (On the open mapping theorem. Seever's theorem.) Rev. Acad. Ci. 75 (1981), 33-37.

[84] Sobre el teorema de la gráfica cerrada. Teorema de BanachSchauder. (On the closed graph theorem. The Banach-Schauder theorem.) Rev. Acad. Ci. 75 (1981), 39-46.

[85] Sobre una extensión de la teoría de De Wilde. (On an extension of De Wilde's theory.) Rev. Acad. Ci. 75 (1981), 579-587.

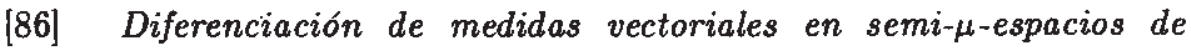
Lusin. (Differentiation of vector measures in semi- $\mu$-spaces of Lusin.) Rev. Acad. Ci. 75 (1981), 1065-1082.

[87] Diferenciación de medidas vectoriales en espacios topológicos. (Differentiation of vector measures in topological spaces.) Rev. Acad. Ci. 75 (1981), 1083-1099.

[88] El teorema de von Staudt y sus generalizaciones. (Von Staudt's theorem and its generalizations.) Volume in honor of Germán Ancochea Quevedo, 31-34. Real Academia de Ciencias and Faculdad de Ciencias Matemáticas, Universidad Complutense, Madrid, 1981 .

[89] Sobre el operador maximal de Hardy-Littlewood y las propiedades de crecimiento. (On the Hardy-Littlewood maximal operator and growth properties.) Rev. Acad. Ci. 76 (1982), 11-20.

[90] (with Pedro Jiménez Guerra) Teorema de Fubini en espacios topológicos arbitrarios. (Fubini's theorem in arbitrary topological spaces.) Rev. Acad. Ci. 76 (1982), 503-518.

[91] Diferenciación de medidas sobre espacios uniformes. (The differentiation of measures on uniform spaces.) Rev. Acad. Ci. 76 (1982), 519-529.

[92] (with Pedro Jiménez Guerra) Strictly localizable measures. Nagoya Math. J. 85 (1982), 81-86. 
[93] Integración de funciones con valores en un espacio localmente convexo respecto de medidas infinitas. (Integration of functions with values in a locally convex space with respect to infinite measures.) Rev. Acad. Ci. 77 (1983), 49-60, 61-78, 529-541, 543-567.

[94] La propiedad de Radon-Nikodym, $\sigma$-dentabilidad y martingalas en espacios localmente convexos II. (The Radon-Nikodym property, $\sigma$-dentability and martingales in locally convex spaces II.) Rev, Acad. Ci. 78 (1984), 59-64.

[95] (with José L. de María) Sobre el corazón de una función vectorial. (On the heart of a vector-valued function.) Rev. Acad. Ci. 78 (1984), 65-75.

[96] Sobre el dual de un espacio localmente convexo con la propiedad de Radon-Nikodym. (On the dual of a locally convex space with the Radon-Nikodym property.) Rev. Acad. Ci. 78 (1984), 77-83, $85-96$.

[97] (with Francisco Domingo) Convergencia en casi todo punto de series de Fourier de funciones con valores en espacios de Banach. Generalizaciones. (The convergence at almost every point of Fourier series of functions with values in Banach spaces. Generalizations.) Rev. Acad. Ci. 78 (1984), 97-107.

[98] La propiedad de Radon-Nikodym del dual fuerte de un espacio infratonelado. (The Radon-Nikodym property of the strong dual of an infrabarrelled space.) Rev. Acad. Ci. 78 (1984), 471-475.

[99] La propiedad de Radon-Nikodym del dual de un espacio localmente convexo. (The Radon-Nikodym property of the dual of a locally convex space.) Rev. Acad. Ci. 78 (1984), 476-484.

[100] Un convexo sin representación integral extremal. (A convex set without extremal integral representation.) Mathematical Contributions in honor of Luis Vigil, 251-255. Zaragoza, 1984.

[101] La propiedad de Dieudonné y la propiedad $V$ de Pelczynski sobre los espacios $C(\Omega, E)$. (Dieudonné's property and Pelczynski's property $V$ on the spaces $C(\Omega, E)$.) Rev. Acad. Ci. 79 (1985), $33-38$. 
[102] (with A. García López) Sobre algunas propiedades de espacios de funciones con valores vectoriales. (On some properties of spaces of vector-valued functions.) Contribuciones Matemáticas. Studies in honor of D. Francisco Botella, 109-116. Madrid, 1986.

[103] (with Fernạndo Bombal) Some classes of operators on $C(K, E)$. Extension and applications. Arch. Math. (Basel) 47 (1986), 5565.

[104] Arquímedes. (Archimedes.) Historia de la Matemática hasta el siglo XVII, 79-99. Real Academia de Ciencias, Madrid, 1986.

[105] Integration of Banach-valued functions. Publicación del Departamento de Análisis Matemático, 107-118. Universidad Complutense, Madrid, 1987.

[106] Extremal integral representation. Proc. Roy. Irish Acad. Sect. A 87 (1987), 45-61.

[107] Funciones asociadas a un conjunto del plano complejo. (Functions associated with a set in the complex plane.) Rev. Acad. Ci. $81(1982), 9-21$.

[108] Influencia de la frontera en la representación conforme. (The influence of the boundary in conformal representation.) Rev. Acad. Ci. 81 (1987), 23-35.

[109] Desigualdades para las derivadas de un polinomio. (Inequalities for the derivatives of a polynomial.) Rev. Acad. Ci. 81 (1987), $467-485$.

[110] Regularización de sucesiones. (The regularization of sequences.) Rev. Acad. Ci. 81 (1987), 455-465.

[111] Desigualdades para las cotas de los desarrollos asintóticos. (Inequalities for the bounds of asymptotic expansions.) Rev. Acad. Ci. 81 (1987), 617--633.

[112] Solución del problema de equivalencia de clases de funciones con desarrallo asintótico. (Solution of the problem of equivalence of classes of functions with an asymptotic expansion.) Rev. Acad. Ci. 81 (1987), 601-615. 
[113] (with Francisco L. Hernández) On $\ell^{p}$-complemented copies in Orlicz spaces. Israel J. Math. 62 (1988), 37-55.

[114] Newton. Historia de la Matemática en los siglos XVII y XVIII, 31-60. Real Academia de Ciencias, Madrid, 1988.

[115] Julio Rey Pastor, el maestro. (Julio Rey Pastor, the teacher.) Conmemoración del Centenario del Nacimiento de D. Julto Rey Pastor (Conmemoration of the Centenary of the birth of D. Julio Rey Pastor), 11-18. Real Academia de Ciencias, Madrid, 1988.

[116] (with Pedro Jiménez Guerra) On the completeness of $L^{\alpha}$ for locally convex spaces. Arch. Math. (Basel) 52 (1989), 82-91.

[117] (with Francisco L. Hernández) On $\ell^{p}$ - complemented copies in Orlicz spaces II. Israel J. Math. 68 (1989), 27-55.

[118] A generalization of a theorem of Albert and applications. Proceedings of the Mathematical Meeting in Honor of A Dou, 191-196. Universidad Complutense, Madrid, 1989.

[119] (with José L. de Maria) Structure of measures on topological spaces. Rev. Mat. Univ. Complut. Madrid 2, número suplementario (1989), 103-118.

[120] (with Francisco L. Hernández) On minimality and $\ell^{p}$. complemented subspaces of Orlicz function spaces. Rev. Mat. Univ. Complut. Madrid 2, número suplementario (1989), 129 136.

[121] Perfect measure spaces and coperfect spaces. Mathematical Contributions in honor of Antonio Plans, 239-249. Zaragoza, 1990.

[122] On the variation of an indefinite integral in Banach spaces. Ann. Soc. Math. Polon. Ser. I Comment. Math. Prace Mat. 30 (1990), $167-170$.

[123] Subspacios de un espacio de Banach con una base incondicional. (Subspaces of a Banach space with inconditional basis.) Homenaje al Prof. Dr. Nácere Hayek Calil, 265-267. La Laguna, 1990. 
[124] Quasi-Radon measures and Radon measures of type $(\mathcal{H})$. Rend. Circ. Mat. Palermo (2) 40 (1991), 142-152.

[125] (with José L. de Maria) The space $\left(\ell_{00} / c_{0}\right.$, weak) is not a Radon space. Proc. Amer. Math. Soc. 112 (1991), 1095-1100.

[126] Banach spaces not having copies of $L_{\infty}$ and Z. Rev. Acad. Ci. $84(1990), 403-407$.

[127] On the structure of a vector measure. Rev. Acad. Ci. 84 (1990), 409-414.

[128] (with Francisco L. Hernández), Orlicz spaces containing singular $\ell^{p}$-complemented copies. Function Spaces (ed. J. Musielak et al.), 15-22. Teubner Texte zur Mathematik, Bd. 120. Teubner, Leipzig, 1991.

[129] Recuerdo a Euler. (A remembrance of Euler.) Historia de la Matemática en el siglo XIX, $1^{a}$ parte, 11-41. Real Academia de Ciencias, Madrid, 1992.

[130] (with José L, de Maria) On measurable sets of a $\tau$-additive measure. Homenaje a Pablo Bobillo Guerrero, 241-259. Universidad de Granada, 1992.

[131] (with Francisco L. Hernández) Remarks on the Orlicz function spaces $L^{\varphi}(0, \infty)$. Math. Nachr. 156 (1992), 225-232.

[132] Strictly localizable measures. Rend. Circ. Mat. Palermo (2) 41 (1992), 295-301.

[133] (with José L. de Maria) On measures on $\sigma$-metrizable spaces. Atti Sem. Mat. Fis. Univ. Modena 41 (1993), 131-147.

[134] (with José L. de Maria) Banach spaces which are Radon spaces with the weak topology. Bull. London Math. Soc. 25 (1993), 577581.

[135] (with José L. de Maria) Cauchy. Historia de la Matemática en el siglo XIX, $2^{a}$ parte, 79-111. Real Academia de Ciencias, Madrid, 1994. 
[136] Cantor y la teoria de conjuntos. (Cantor and the theory of sets.) Historia de la Matemática en el siglo XIX, $2^{a}$ parte, 301-317. Real Academia de Ciencias, Madrid, 1994.

[137] On the complemented subspaces of $c_{0}(I)$ and $\ell_{p}(I)$ for $1<p<\infty$. Atti. Sem. Mat. Fis. Univ. Modena 42 (1994), 399-402.

[138] On the uniqueness of non-locally finite invariant measures on a topological group. J. Math. Anal. Appl 188 (1994), 387-397.

[139] (with Francisco L. Hernández)Lattice-embedding $L^{p}$ into Orlicz spaces. Israel J. Math. 90 (1995), 167-188.

[140] (with José L. de Maria) Weakly $\theta$-refinable spaces and $\tau$-additive measures. Mathematical Contributions. Libro-homenaje al Profesor D. José Javier Etayo Miqueo, 203-207.

[141] Teoría de la medida en espacios métricos y topológicos. (The theory of measure in metric and topological spaces.) Publicaciones del Departamento de Análisis Matemático, Sección 1, no. 29. Universidad Complutense, Madrid. 243 pp.

[142] (with Pedro Jiménez Guerra) A general solution of the MongeKantorovich mass-transfer problem. J. Math. Anal. Appl., 202 (1996), 492-510.

[143] Subspaces of Banach lattices. Preprint.

[144] (with José L. De Maria) On measures on metric spaces. Preprint.

[145] (with José L. de Maria) On measures on Banach spaces with the weak topology. Preprint.

[146] On superbarrelled spaces. Closed graph theorems. Preprint.

[147] Subspaces with symmetric basis in Orlicz space $\ell^{F}(I)$. Preprint.

[148] Sobre el espacio $\mathcal{D}^{\prime}(1)$ de distribuciones secuenciales. (On the space $\mathcal{D}^{\prime}(1)$ of sequential distributions.) Preprint. 
Department of Mathematics

Recibido: 23 de Mayo de 1996 University of Maryland

College Park, Maryland 20742

U.S.A 